\title{
Discontinuous modelling of shear bands using adaptive meshfree methods
}

\author{
Rabczuk T. \\ University of Canterbury, Department of Mechanical Engineering, Private Bag \\ 4800, Christchurch, New Zealand \\ Samaniego E. \\ Civil Engineering School, Universidad de Cuenca, Avda. 12 de abril, s/n, Cuenca, \\ Ecuador
}

\begin{abstract}
A simple methodology to model shear bands as strong displacement discontinuities in an adaptive meshfree method is presented. The shear band is represented by a displacement jump at discrete particle positions. The displacement jump in normal direction is suppressed with penalty method. Loss of material stability is used as transition criterion from continuum to discontinuum. The method is twoand three-dimensional. Examples of complicated shear banding including transition from brittle-to-ductile failure are studied and compared to experimental data and other examples from the literature.
\end{abstract}

Key words: meshfree methods; shear bands; loss of hyperbolicity; traction-separation laws

\section{INTRODUCTION}

A shear band can be described as a narrow region in a solid undergoing intense shearing. After a shear band is fully developed, relative sliding of the two sides of the band similar to mode II fracture can be observed. Shear bands can be regarded as specific instances of the more general phenomenon of strain localization and as material instabilities.

Email addresses: Timon.Rabczuk@canterbury.ac.nz (Rabczuk T.), Esteban.Samaniego@host.ucuenca.edu.ec (Samaniego E.). 
When dealing with rate independent materials undergoing strain softening, the development of bands of localized deformation has classically been linked to the loss of hyperbolicity of the linearized Initial Boundary Value Problem (IBVP). In Bazant and Belytschko [10], a closed form solution was obtained for a wave propagation problem in a one-dimensional bar. It was shown that, despite the loss of positivity of the tangent modulus, a solution could be obtained if the region undergoing strain softening was restricted to a set of (Lebesgue) measure zero. However, the solution was considered to be meaningless from a physical point of view, because it implied zero dissipation. As pointed out by Armero [4], for example, this shows the necessity of including some dissipative mechanism in the region in which hyperbolicity has been lost.

The above discussion highlights two crucial phases of the mathematical modelling of strain localization:

1) The detection of loss of material stability.

2) The adoption of some model to capture the post-localization behavior.

The former determines the material points that belong to a localization band, whereas the latter enables one to obtain physically meaningful solutions after the onset of localization.

Although numerous studies have been pursued to limit the localization by regularization procedures or localization limiters (as in Lasry and Belytschko [50], Needleman and Tvergaard [63]; see Jirasek [45] for a review), regularization does not overcome the basic difficulty that the scale of a shear band is much smaller than the scale of structure. For example, in a $1 \mathrm{~m}$ structure the width of a shear band may be $10^{-5} \mathrm{~m}$, and its structure is quite rich, so 10 to 20 low order elements are needed to adequately resolve its morphology and evolution. Thus a brute force approach to this problem is not viable. An alternative approach employed here is to model shear bands as discontinuities.

Many numerical methods were developed to model discontinuities such as cracks and shear bands. One of the most popular classes of methods are the interelement separation models, see [86], Camacho and Ortiz [24], Ortiz et al. [69], Zhou and Molinari [89] that were initially developed for cracks and later on extended to shear bands, Yang et al. [88]. In these methods, cracks or shear bands are only allowed to develop along existing interelement edges. This provides the method with comparative simplicity, but can result in an overestimate of the dissipated energy when the actual discontinuity paths are not coincident with element edges. Mesh sensitivity has been reported, see Falk et al. [33]; this sensitivity can be mollified by adding randomness to the strength, as in Zhou and Molinari [89].

The extended finite element method is a method that allows the crack or shear 
band to propagate arbitrarily. Methods for static crack problems in two and three dimensions were developed by Moes and Belytschko [59], Moes et al. [60] and Gravouil et al. [37], respectively. The method was applied to dynamic crack problems in Belytschko et al. [13] and to dislocations in Ventura et al. [80]. A recent paper applies the extended finite element method to discontinuous modelling of shear bands, Samaniego and Belytschko [77]. However, these methods require an explicit representation of the crack or shear band surface, which usually has been provided by level sets. This makes the treatment of phenomena such as branching more difficult, since additional level sets need to be introduced whenever a branch occurs. Another method that does not need a surface representation of the crack or shear band is the embedded discontinuity model (Belytschko et al. [15], Armero and Garikipati [5], Oliver et al. [65]).

Shear bands in meshfree methods have usually been modelled without special treatment other than refinement around the expected paths, see e.g. Hao et al. [39, 40, 41], Li et al. [53, 54]. Cracks in meshfree methods have been treated by Krysl and Belytschko [49] and Organ et al. [68].

In a recent paper, Rabczuk and Belytschko [72] introduced a 'cracking particle' method for modelling arbitrary crack propagation in two dimensions. In [74], the method was extended to three dimensions. In this paper, we will extend the approach to model shear bands in metals. To supress normal crack opening, that is usually not allowed in shear bands, a penalty is introduced. Furthermore, h-adaptivity is used around the shear band tip to guarantee smooth shear band paths. As shown for cracks in [74] for cracks, the quality of the results depend on the accuracy around the crack tip and h-adaptivity is ideally suited for meshfree methods.

The paper is organized as follows. First, the approximation of the displacement field is described. The IBVP with dissipate interface is stated. The constitutive models are given next. First, we describe the continuum model before discussing the interface model. The use of the local hyperbolicity or stability for a rate-dependent material, respectively, of the momentum equation as a criterion of material stability is described. A stress projection method is proposed that enforces traction continuity normal to the discontinuity. The discretization of the linear momentum equation is derived in section 5. Results for a variety of problems compared to experimental results are given in section 7 . 


\section{DISPLACEMENT FIELD}

Consider a displacement field which is continuous in the entire domain except at the shear band where the displacement is discontinuous. As e.g. in Belytschko and Black [12], Dolbow et al. [30], Moes et al. [60], the displacement is decomposed into continuous and discontinuous parts :

$$
\mathbf{u}(\mathbf{X}, t)=\mathbf{u}^{\text {cont }}(\mathbf{X}, t)+\mathbf{u}^{e n r}(\mathbf{X}, t)
$$

where $\mathbf{X}$ are the material coordinates, $t$ is the time, $\mathbf{u}^{\text {cont }}$ denotes the continuous displacement and $\mathbf{u}^{e n r}$ the discontinuous part, also called the enrichment.

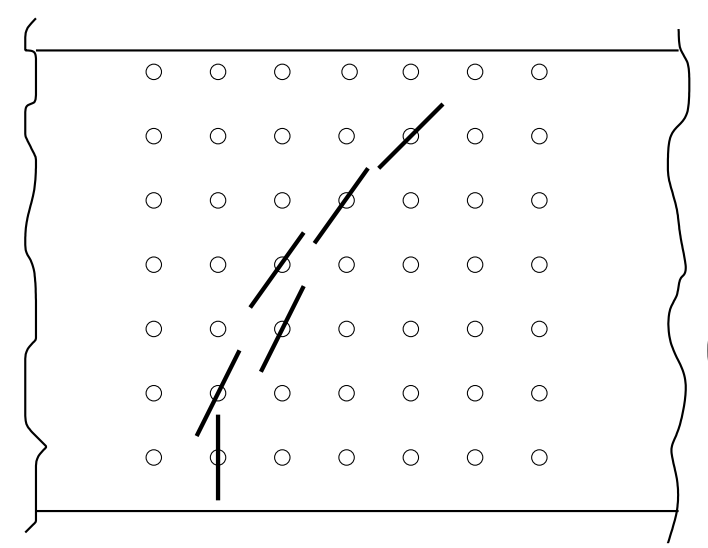

a)

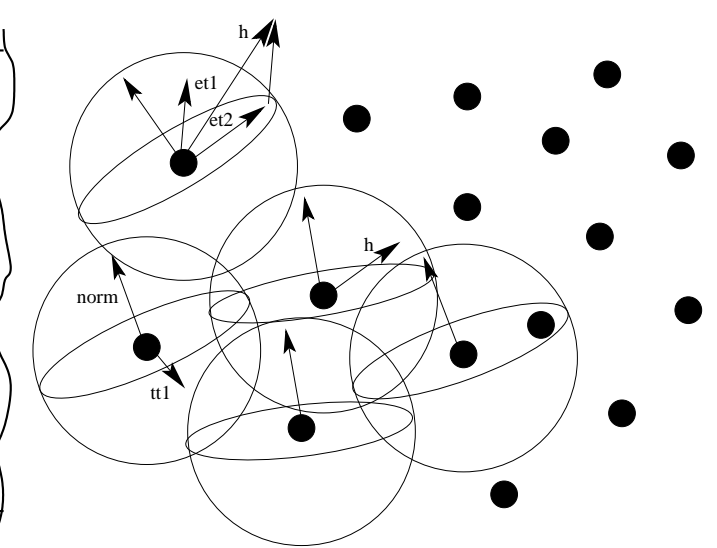

(b)

Fig. 1. Schematic of the shear band model for two and three dimensions

The shear band is modelled by a set of discrete cohesive segments that cross the corresponding particle as shown in figure 1 . The advantage of this approach is that no crack path continuity is required and hence complicated shear band patterns can be described quite simply and naturally.

Let $\mathcal{N}$ be the total set of nodes in the model and $\mathcal{N}_{c}$ the set of cracked nodes. To model the discontinuous part of the displacement, the test and trial functions are enriched with sign functions which are parametrized by $\delta \mathbf{q}_{I}$ and $\mathbf{q}_{I}$, respectively:

$$
\begin{gathered}
\mathbf{u}^{h}(\mathbf{X}, t)=\sum_{I \in \mathcal{N}} \Phi_{I}(\mathbf{X}) \mathbf{u}_{I}(t)+\sum_{I \in \mathcal{N}_{c}} \Phi_{I}(\mathbf{X}) S\left(f_{I}(\mathbf{X})\right) \mathbf{q}_{I}(t) \\
\delta \mathbf{u}^{h}(\mathbf{X})=\sum_{I \in \mathcal{N}} \Phi_{I}(\mathbf{X}) \delta \mathbf{u}_{I}+\sum_{I \in \mathcal{N}_{c}} \Phi_{I}(\mathbf{X}) S\left(f_{I}(\mathbf{X})\right) \delta \mathbf{q}_{I}
\end{gathered}
$$

where $f_{I}(\mathbf{X})$ is given by

$$
f_{I}(\mathbf{X})=\mathbf{n}_{0} \cdot\left(\mathbf{X}-\mathbf{X}_{I}\right)
$$


where $\mathbf{n}_{0}$ is the normal to the crack in the reference configuration and $\mathbf{u}_{I}$ are the particle displacements. Note, that we will later on use an updated Lagrangian version and describe all quantities in the current configuration. However, the principle stays the same. The normal $\mathbf{n}$ in the current configuration is obtained from a material stability analysis that will be explained later. To enforce the shear band not to open, a penalty is introduced. This will be described later as well.

The sign function $S(\xi)$ is defined as:

$$
S(\xi)=\left\{\begin{array}{c}
1 \quad \forall \xi>0 \\
-1 \quad \forall \xi<0
\end{array}\right.
$$

We will employ first-order complete element free Galerkin method, i.e. with linear polynomial basis; for more details, see e.g. Belytschko et al. [16], Belytschko and Lu [18], Belytschko et al. [19], Lu et al. [56]. For most computations, it is sufficient to use a Lagrangian kernel. When the deformations become too large, we will switch to an Eulerian kernel. Discretizations based on Eulerian kernel tend to have difficulties to exactly represent the onset of material failure, see Belytschko and Xiao [21], Xiao and Belytschko [85]. However, for large distortions of the image of the domain of influence in the current configuration, Lagrangian kernels tend to become instable. The methodology of switching from Lagrangian to Eulerian kernel is described in detail in [74].

The gradient of the test functions with respect to the material coordinates is given by

$$
\begin{aligned}
\nabla_{0} \delta \mathbf{u}(\mathbf{X}) & =\sum_{I \in \mathcal{N}} \nabla_{0} \Phi_{I}(\mathbf{X}) \otimes \delta \mathbf{u}_{I}+\sum_{I \in \mathcal{N}_{c}} \nabla_{0} \Phi_{I}(\mathbf{X}) S\left(f_{I}(\mathbf{X})\right) \otimes \delta \mathbf{q}_{I} \\
& +\sum_{I \in \mathcal{N}_{c}} \Phi_{I}(\mathbf{X}) \nabla_{0} S\left(f_{I}(\mathbf{X})\right) \otimes \delta \mathbf{q}_{I}
\end{aligned}
$$

The last term on the RHS of eq. (6) is dropped since $\Omega_{0}$ is treated as an open set with the crack as part of the boundary and $\nabla_{0} S\left(f_{I}(\mathbf{X})\right)$ is nonzero only on $\Gamma^{c}$. The trial functions have an identical structure.

\section{IBVP WITH A DISSIPATIVE INTERFACE. WEAK FORM}

Consider a solid $\Omega$ with material points $\mathbf{X}$. Its boundary $\Gamma$ is partitioned into two subsets, $\Gamma^{t}$ and $\Gamma^{u}$, upon which tractions and displacements are applied. The internal surface of discontinuity is approximated by local disctontinuities

$\Gamma_{I}^{c}$, where cohesive forces act, see figure 1 . 
In the quasi-static case, the solution of a Boundary Value Problem (BVP) with internal boundaries is to be sought in the so-called Space of Functions with Bounded Variations (BV) (see Evans and Gariepy [31]). This space contains functions whose distributional gradients are bounded (Radon) measures. Within this functional framework, discontinuities in the displacement field can be properly treated. For the dynamical case, it seems reasonable to assume that the restriction of the solution to a given instant of time also belongs to $B V$.

We will consider for a given time $t$ the space $S$ of piecewise suitably smooth functions. Let us also define the corresponding space of test functions as

$$
V:=\{\delta \mathbf{u} ; \mathbf{u}+\delta \mathbf{u} \in S, \forall \mathbf{u} \in S\}
$$

Define $\Gamma^{c}=\bigcup \Gamma_{I}^{c}$. Now, we state the following weak form of an IBVP for a solid with internal dissipative interfaces $\Gamma_{I}^{c}$ :

GIVEN the initial displacement, $\mathbf{u}_{0}$, and the initial stress, $\boldsymbol{\sigma}_{0}$,

FIND $\mathbf{u} \in S$ for every time $t$ in the time interval of analysis

SUCH THAT, for all $\delta \mathbf{u} \in V$,

$$
\begin{aligned}
\int_{\Omega} \rho \delta \mathbf{u} \cdot \ddot{\mathbf{u}} d \Omega & =\int_{\Omega_{0} \backslash \Gamma_{0}^{c}}(\nabla \otimes \delta \mathbf{u}): \boldsymbol{\sigma} d \Omega_{0}-\int_{\Omega} \delta \mathbf{u} \cdot \mathbf{b} d \Omega-\int_{\Gamma^{t}} \delta \mathbf{u} \cdot \mathbf{t} d \Gamma \\
& +\int_{\Gamma^{c}} \delta \llbracket \mathbf{u} \rrbracket \cdot \mathbf{t}^{C} d \Gamma+\int_{\Gamma^{c}} \alpha \llbracket \mathbf{u}_{n} \rrbracket \delta \llbracket \mathbf{u}_{n} \rrbracket d \Gamma
\end{aligned}
$$

in the time interval of the analysis, where $\rho$ is the mass density and $\boldsymbol{\sigma}$ is the Cauchy stress. The last term on the right hand side penalizes the appearance of a discontinuity in the direction normal to the discontinuity segment. The subindex $n$ denotes the component normal to the cohesive segment.

\section{CONSTITUTIVE MODELS}

\subsection{Constitutive model}

The following subsection will describe the continuum constitutive models used in our computation. 


\subsubsection{Continuum model 1: J2-plasticity}

The constitutive behavior in $\Omega \backslash \Gamma^{c}$ is governed by a continuum constitutive model, whereas the material description on $\Gamma^{c}$ is governed by a tractionseparation model (often called a cohesive law). Similar approaches to the modelling of shear bands can be found in Olmstead et al. [67].

For the continuum model, a $J_{2}$ plasticity model for moderately large strains with an isotropic hardening/softening law is considered. We employ the logarithmic spin and the Hencky srain, which assure perfect reversibility and therefore zero dissipation in a closed cycle for elastic-only processes (c.f. $[55,76]$ ). We assume that material stability is not an issue in the elastic-only regime (this issue is discussed in reference [23]).

If we additively decompose the spatial velocity gradient $\boldsymbol{l}=\boldsymbol{\nabla} \boldsymbol{v}$ into its symmetric (strain rate, $\dot{\boldsymbol{\varepsilon}}$ ) and skew-symmetric (spin, $\boldsymbol{\Omega}$ ) parts, it is possible to obtain the following relation between the strain rate and the logarithmic corotational rate of the spatial Hencky strain tensor (c.f. [76]):

$$
\dot{\varepsilon}=\frac{\circ}{\ln (\boldsymbol{V})}
$$

where $\boldsymbol{V}$ is the left stretch tensor and

$$
\frac{\circ}{\ln (\boldsymbol{V})}=\frac{\dot{ }}{\ln (\boldsymbol{V})}+\ln (\boldsymbol{V}) \boldsymbol{W}^{\log }-\boldsymbol{W}^{\log } \ln (\boldsymbol{V})
$$

where the superimposed dot indicates a time derivative and

$$
\boldsymbol{W}^{\log }=\boldsymbol{\Omega}+\sum_{i=1}^{3} \sum_{j \neq i=1}^{3} h_{\log }\left(\frac{\lambda_{i}^{2}}{\lambda_{j}^{2}}\right)\left(\boldsymbol{n}_{i}^{b} \otimes \boldsymbol{n}_{i}^{b}\right) \underbrace{\frac{\circ}{\ln (\boldsymbol{V})}}_{\dot{\boldsymbol{\varepsilon}}}\left(\boldsymbol{n}_{j}^{b} \otimes \boldsymbol{n}_{j}^{b}\right)
$$

where $\lambda_{i}$ (the principal stretches) and $\boldsymbol{n}_{i}^{b}$ are obtained from the spectral decomposition of the right Cauchy-Green tensor $(\boldsymbol{b})$ as:

$$
\boldsymbol{b}=\sum_{i=1}^{3} \lambda_{i}^{2} \boldsymbol{n}_{i}^{b} \otimes \boldsymbol{n}_{i}^{b}
$$

The skew-symmetric function $h_{l o g}(z)$ is written, for $z>0$, as:

$$
h_{\log }(z)=\left\{\begin{array}{c}
\frac{1+z}{1-z}+\frac{2}{\ln (z)} \quad z \neq 1 \\
0 \quad z=1
\end{array}\right.
$$


Introducing the proper orthogonal tensor $\boldsymbol{R}^{\log }$ generated by $\boldsymbol{W}^{\log }$ :

$$
\dot{\boldsymbol{R}}^{\log } \boldsymbol{R}^{\log T}=\boldsymbol{W}^{\log }
$$

we can obtain the two tensors involved in the constitutive equations; the corotational stress tensor:

$$
\hat{\mathbf{T}}^{\log }=\boldsymbol{R}^{\log T} \boldsymbol{\tau} \boldsymbol{R}^{\log }
$$

where $\boldsymbol{\tau}$ is the Kirchhoff stress tensor, and the corresponding work-conjugate strain rate:

$$
\hat{\boldsymbol{D}}=\boldsymbol{R}^{\log T} \frac{\circ}{\ln (\boldsymbol{V})} \boldsymbol{R}^{\log }
$$

The rotated strain rate is assumed to be additively decomposable into elastic $\left(\boldsymbol{D}^{e}\right)$ and plastic $\left(\boldsymbol{D}^{p}\right)$ parts:

$$
\hat{D}=\hat{D}^{e}+\hat{D}^{p}
$$

The state equation for the stress rate, assuming isotropic elasticity, reads

$$
\hat{\dot{T}}=\hat{\mathbf{C}}:\left(\hat{D}-\hat{D}^{p}\right)
$$

The stress-like internal variable is assumed to be a function of the equivalent plastic strain, so

$$
g=\mathrm{g}\left(\varepsilon^{*}\right)
$$

$g$ denotes the stress-like internal variable and $\varepsilon^{*}$ the equivalent plastic strain. The evolution equation for the plastic strain is

$$
\boldsymbol{D}^{p}=\gamma \frac{\partial f}{\partial \hat{\boldsymbol{T}}}=\gamma \mathbf{m}
$$

where $f$ is the yield function (which defines the elastic region in the generalized stress space) and $\gamma$ is the plastic multiplier, as well as an evolution equation for the equivalent plastic strain,

$$
\dot{\varepsilon}^{*}=\gamma \frac{\partial f}{\partial g}
$$

The yield function is defined by

$$
f(\hat{\boldsymbol{T}}, g)=\bar{\Upsilon}(\hat{\boldsymbol{T}})-g
$$

where $\bar{\Upsilon}$ is some scalar valued function of the stress state $\hat{\mathbf{T}}$. Function (19) defines the set of admissible states as those for which

$$
f(\hat{\boldsymbol{T}}, g) \leq 0
$$


Table 1

Elasticity-consistent algorithm for elasto-plastic finite strain problems

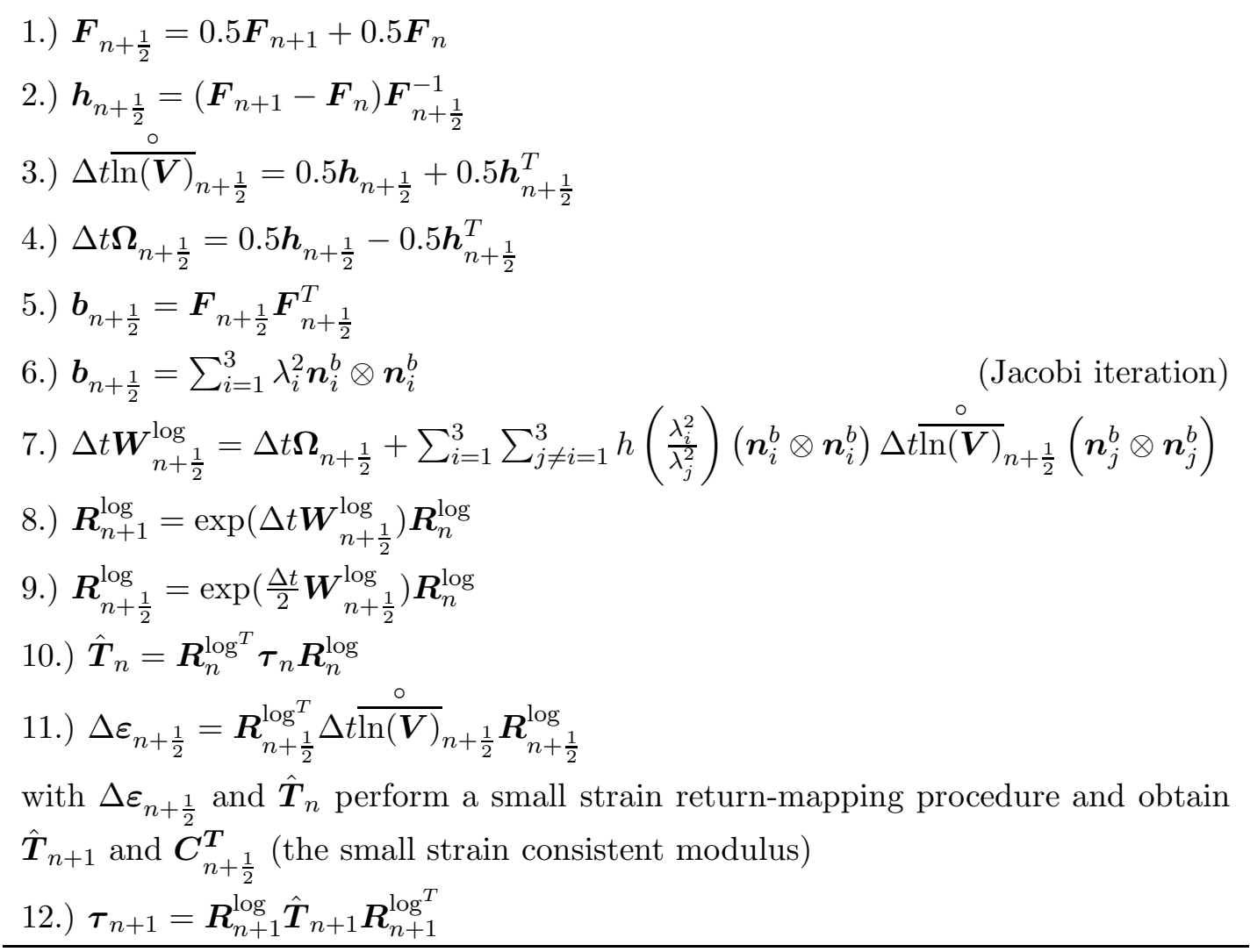

Finally, we have the loading/unloading complementary conditions,

$$
\gamma \geq 0 ; f(\hat{\boldsymbol{T}}, g) \leq 0 ; \gamma f(\hat{\boldsymbol{T}}, g)=0
$$

and the plastic consistency condition,

$$
\gamma \dot{f}(\hat{\boldsymbol{T}}, g)=0
$$

The algorithm is depicted in table 1; it uses the mid-point rule and the exponential map (e.g. [2]) for the update of the logarithmic rotation. It is interesting to note that only internal variables and the deformation gradient are required to carry out the constitutive update. The term $\Delta t$ represents the time increment.

\subsection{Continuum model 2: Johnson-Cook model}

The Johnson-Cook model [46] with $J_{2}$ plasticity includes strain rate and temperature effects. The effective yield stress of the Johnson-Cook model is given by 


$$
\sigma_{Y}=\left(A+B \gamma^{n}\right)\left(1+C \ln \dot{\epsilon}^{*}\right)\left(1-T^{*}\right)
$$

with $\dot{\epsilon}^{*}=\dot{\gamma} / \dot{\gamma}_{0}$ where $\gamma$ is the effective plastic strain and $\dot{\gamma}_{0}$ is the reference strain rate taken to be $1.0 / \mathrm{s}$ and

$$
T^{*}=\frac{T-T_{r}}{T_{m}-T_{r}}
$$

where $T_{r}$ is the reference temperature and $T_{m}$ is the melting temperature. We assume that the plastic deformation is completely transformed into heat, so $\beta=1$ for the temperature update:

$$
\Delta T=\int_{0}^{\gamma} \frac{\beta}{\varrho c_{v}} \sigma_{Y} d \gamma
$$

\subsection{Perzyna viscoplastic model}

Within our examples, we used a Perzyna viscoplastic model as in Diez et al. [28]. We briefly describe the model; for more details, see Diez et al. [28]. Basic assumption is the decomposition of the strain tensor into elastic and viscoplastic part:

$$
\boldsymbol{\epsilon}=\boldsymbol{\epsilon}^{e}+\boldsymbol{\epsilon}^{v p}
$$

where the superscript $e$ and $v p$ denotes the elastic and visco-plastic part, respectively. The stress rate is given by

$$
\dot{\boldsymbol{\sigma}}^{\nabla}=\mathbf{C}^{t}:\left(\dot{\boldsymbol{\epsilon}}-\dot{\boldsymbol{\epsilon}}^{v p}\right)
$$

where $\mathbf{C}^{t}$ is the tangent stiffness and $\nabla$ indicates Lee derivatives. For associated visco-plasticity, the visco-plastic strain rate is, Perzyna [70]:

$$
\dot{\boldsymbol{\epsilon}}^{v p}=\gamma\left(\frac{<f>}{\overline{\sigma_{0}}}\right)^{N} \frac{\partial f}{\partial \boldsymbol{\sigma}}
$$

where $<>$ are the Macauley brackets, $\sigma_{0}$ is the initial yield stress and $\gamma$ and $N$ are material parameters. The yield stress $\bar{\sigma}$ is assumed to depend linearly on the equivalent visco-plastic strain $\kappa$ :

$$
\bar{\sigma}=<\overline{\sigma_{0}}+h \kappa>
$$

with hardening modulus $h$. If $h<0$, we are talking about softening modulus. 


\section{SHEAR BAND MODEL}

\subsection{Shear band initiation}

Once an inviscid material loses stability (in a sense that will be made clear later), the standard governing equations are no longer applicable, because they do not model the correct dissipation. Several remedies to this problem have been proposed. One of them is to include a dissipative mechanism once the material has lost stability, see Belytschko et al. [13], Wang et al. [83].

Within the context of nonlinear fracture mechanics, these dissipative mechanisms have been introduced by means of the so-called cohesive surfaces. We adopt a similar methodology here for modelling shear bands.

Shear bands have been shown to be a consequence of softening in Clifton et al. [27] and Wright and Walter [84]. In a rate-dependent material, the PDE does not change type and only undergoes a material instability, whereas in a rate independent material, it loses hyperbolicity. Note that loss of material stability and loss of hyperbolicity is checked by the same criterion. A classical definition of material stability is the Legendre-Hadamard condition, which establishes that for any nonzero vectors $\mathbf{n}$ and $\mathbf{h}$ the following point-wise inequality must hold for material stability:

$$
(\mathbf{n} \otimes \mathbf{h}): \mathbf{A}:(\mathbf{n} \otimes \mathbf{h})>0 \forall \mathbf{n} \text { and } \forall \mathbf{h}
$$

where $\mathbf{A}$ is related to the constitutive tangent operator for the Truesdell rate, see Belytschko et al. [17]:

$$
\mathbf{A}=\mathbf{C}+\boldsymbol{\sigma} \otimes \mathbf{I}
$$

The vector $\mathbf{n}$ defines the direction of propagation and $\mathbf{h}$ describes the localization direction. For a mode I-crack, $\mathbf{h}$ is parallel to $\mathbf{n}$; for mode II or a shear band, $\mathbf{h}$ is perpendicular to $\mathbf{n}$. This should be the outcome of the bifurcation analysis and can be used as criterion for brittle-to-ductile failure.

Based on (30), let us define for a given material point of a solid at a given time, the acoustic tensor

$$
\mathbf{Q}=\mathbf{n} \cdot \mathbf{A} \cdot \mathbf{n}
$$

Equation (30) is equivalent to the condition that the minimum eigenvalue of $\mathbf{Q}$ is strictly positive, so we say a material point is stable whenever the minimum eigenvalue of $\mathbf{Q}$ is strictly positive and unstable otherwise. 


\subsection{Interface model}

The displacement jump in tangential direction is given by

$$
[[u]]_{T}=\mathbf{h} \cdot[[\mathbf{u}]]
$$

and in normal direction

$$
[[u]]_{n}=\mathbf{n} \cdot[[\mathbf{u}]]
$$

with

$$
[[u]]_{b}=\sqrt{[[u]]_{T}^{2}+[[u]]_{n}^{2}}
$$

where unloading/reloading is distinguished from loading by the following condition:

$$
\begin{gathered}
\left|[[u]]_{b}\right|^{n+1}>\left|[[u]]_{b}\right|^{n}>\left|[[u]]_{b}\right|^{\text {max }} \text { loading } \\
\left|[[u]]_{b}\right|^{n+1}>\left|[[u]]_{b}\right|^{n} \leq\left|[[u]]_{b}\right|^{\text {max }} \text { reloading } \\
\left|[[u]]_{b}\right|^{n+1} \leq\left|[[u]]_{b}\right|^{n} \text { unloading }
\end{gathered}
$$

where the superscript $n$ indicates the time step. Accordingly, we obtain the velocity jump:

$$
\begin{aligned}
& {[[\dot{u}]]_{T}=\mathbf{h} \cdot[[\dot{\mathbf{u}}]]} \\
& {[[\dot{u}]]_{n}=\mathbf{n} \cdot[[\dot{\mathbf{u}}]]}
\end{aligned}
$$

The cohesive law can be separated into normal and tangential components with respect to the shear band. In tangential direction, the cohesive law is given by

$$
\dot{t}_{T}^{\nabla}=c \llbracket \dot{u} \rrbracket_{T} \quad \text { on } \Gamma^{c}
$$

where $c$ is the softening modulus. In normal direction, we use a penalty to close the crack:

$$
\dot{t}_{n}^{\nabla}=-\alpha \llbracket \dot{u} \rrbracket_{n} \quad \text { on } \Gamma^{c}
$$


where $\alpha$ is the penalty parameter necessary to close the crack in the next time step and the superimposed $\nabla$ indicates Lee derivatives. Crack closure is also enforced with the penalty method. Note, that we won't suppress jumps in the normal direction in case $\mathbf{n}$ is not perpendicular to $\mathbf{h}$ when we have mixed mode failure.

\subsection{Stress continuity condition and stress projection}

Though stress continuity is fulfilled in a variational formulation in a weak sense, it can sometimes be advantageous to enforce it in a strong sense. The use of the projection to impose traction continuity should, in principle, increase the accuracy of the results, because it is imposed in strong form. The approach presented here is also kinematically optimal in the sense that the discontinuity in the displacement field is directly introduced in the approximation space. Furthermore, this stress projection method does not only ensure traction continuity but also symmetry, see Areias et al. [3], Oliver et al. [66] and references therein.

Areias et al. [3] showed that stress continuity can be fulfilled by modifying the constitutive stress $\boldsymbol{\sigma}^{C}$ by the introduction of a symmetric tensor $\mathbf{K}$

$$
\begin{aligned}
\boldsymbol{\sigma} & =\boldsymbol{\sigma}^{C}+h \mathbf{K} \\
\mathbf{t}^{c} & =h \boldsymbol{\sigma}^{C} \cdot \mathbf{n}+h \mathbf{K} \cdot \mathbf{n} \\
\mathbf{e}_{1 T} \cdot \boldsymbol{\sigma} \cdot \mathbf{e}_{1 T}=\mathbf{e}_{1 T} \cdot \boldsymbol{\sigma}^{C} \cdot \mathbf{e}_{1 T} & \Longleftrightarrow \mathbf{e}_{1 T} \cdot \mathbf{K} \cdot \mathbf{e}_{1 T}=0 \\
\mathbf{e}_{1 T} \cdot \boldsymbol{\sigma} \cdot \mathbf{e}_{2 T}=\mathbf{e}_{1 T} \cdot \boldsymbol{\sigma}^{C} \cdot \mathbf{e}_{2 T} & \Longleftrightarrow \mathbf{e}_{1 T} \cdot \mathbf{K} \cdot \mathbf{e}_{2 T}=0 \\
\mathbf{e}_{2 T} \cdot \boldsymbol{\sigma} \cdot \mathbf{e}_{2 T}=\mathbf{e}_{2 T} \cdot \boldsymbol{\sigma}^{C} \cdot \mathbf{e}_{2 T} & \Longleftrightarrow \mathbf{e}_{2 T} \cdot \mathbf{K} \cdot \mathbf{e}_{2 T}=0
\end{aligned}
$$

where $\mathbf{e}_{T 1}$ and $\mathbf{e}_{T 2}$ are two arbitrary vectors perpendicular to $\mathbf{n}$ that fulfill the condition $\mathbf{h}=\mathbf{e}_{T 1}+\mathbf{e}_{T 2}$. The second equation represents the stress continuity condition for all particles which fall in the support size of a cracked/sheared particle. The last three conditions ensure that only stresses in the shear plane and no stresses normal to the plane are transmitted. The constitutive stresses $\boldsymbol{\sigma}^{C}$ are obtained by the constitutive model at the corresponding particle or stress point and $\mathbf{t}^{c}$ denote the cohesive forces at the crack which are approximated with the shape functions at the corresponding particle. We final obtain the following system of equations to obtain $\mathbf{K}$ : 


$$
\left\{\begin{array}{c}
\mathbf{t}^{c}-\boldsymbol{\sigma}^{C} \cdot \mathbf{n} \\
0 \\
0 \\
0
\end{array}\right\}=\left[\begin{array}{cccccc}
n_{1} & 0 & 0 & n_{2} & n_{3} & 0 \\
0 & n_{2} & 0 & n_{1} & 0 & n_{3} \\
0 & 0 & n_{3} & 0 & n_{1} & n_{2} \\
e_{1}^{2} & e_{2}^{2} & e_{3}^{2} & 2 e_{1} e_{2} & 2 e_{1} e_{3} & 2 e_{2} e_{3} \\
t_{1} e_{1} & t_{2} e_{2} & t_{3} e_{3} & t_{1} e_{2}+e_{2} t_{1} & t_{1} e_{3}+e_{1} t_{3} & t_{2} e_{3}+e_{2} t_{3} \\
t_{1}^{2} & t_{2}^{2} & t_{3}^{2} & 2 t_{1} t_{2} & 2 t_{1} t_{3} & 2 t_{2} t_{3}
\end{array}\right]\left\{\begin{array}{c}
K_{11} \\
K_{22} \\
K_{33} \\
K_{12} \\
K_{13} \\
K_{23}
\end{array}\right\}
$$

where $e_{i}, i=1,3$ and $t_{i}, i=1,3$ are the components of $\mathbf{e}_{1 T}$ and $\mathbf{e}_{2 T}$, respectively.

A simpler method to ensure traction continuity in time can be adopted from applying appropriate traction-free boundary conditions in collocation based meshfree methods. In these methods, the stress tensor is rotated into a local coordinate system where the boundary forms the in-plane coordinate axis. The stresses normal to the boundary surface are then set to zero and the stress tensor is rotated back into the global coordinate system.

The same concept is applicable for cohesive cracks. The stress tensor is then rotated into a local crack coordinate system where the crack plane, determined by its normal, spans two coordinate axes of the local coordinate system. The tractions normal to the crack can then be enforced and the stress tensor is afterwards rotated back.

\subsubsection{Dissipation energy}

One difficulty when modelling the post-localization behavior is the correct assumption for the fracture energy. For cracks, the fracture energy is an important material parameter which determines how much energy is dissipated in the crack opening process. For many brittle materials there exists a large amount of literature about values of fracture energy as e.g. the Eur [1] for concrete. However, for shear bands, the determination of a corresponding material parameter is hardly available. It is further not clear that shear bands can always be accurately modelled by a discontinuity surface. Grady [36] and Minnaar and Zhou [58] suggests a general procedure for computing the dissipation energy. They give the dissipation energy as:

$$
G_{f}=\frac{\varrho c_{v}}{\alpha}\left(\frac{9 \varrho^{3} c_{v}^{2} \chi^{3}}{\sigma_{y}^{3} \alpha^{2} \dot{\gamma}}\right)^{1 / 4}
$$

where $\varrho$ is the density, $\chi$ is the thermal diffusion coefficient, $\sigma_{y}$ is the flow stress at strain $\dot{\gamma}_{y}$ and $c_{v}$ is the specific heat. Since eq. (43) takes into account the entire plastic energy though in the cohesive law, an eventually large 
amount of the energy is dissipated before, the portion of the dissipation energy for the cohesive law has to be extracted from eq. (43). Otherwise, it will be overestimated.

\section{DISCRETIZATION}

\subsection{Locking}

Since we use $J_{2}$ plasticity (recall that the material is quasi- incompressible), we expect problems due to locking. Locking in finite elements has always been a concern since its early development. It is well known that bilinear finite elements lock in some problems and that biquadratic elements show a better behavior. In finite elements, there are many possibilities to prevent or at least attenuate locking such as the enhanced strain method (Simo and Armero [79]), the mean-dilatation technique of Nagtegaal et al. [61], the closely related B-bar technique of Hughes [44] and reduced integration technique with stabilization as suggested e.g. in Belytschko and Bindemann [11], Belytschko and Ong [20], Bonet and Bhargava [22]. Recently, mixed/enhanced strain elements and enhanced strain/B-bar elements were proposed by Piltner and Taylor [71] and Cesar de Sa et al. [25].

The picture in meshfree methods is quite different. Even at the end of the 90's, it was claimed that meshfree methods do not exhibit locking, see e.g. Zhu and Atluri [91]. It was claimed that increasing the support size will prevent locking. Studies by Dolbow and Belytschko [29] and Huerta and Fernandez-Mendez [42] have shown that this is not true. Locking can be attenuated but not prevented completely. Huerta and Fernandez-Mendez [42] showed that the number of non-physical locking modes is independent of the support size. Furthermore, they found out that an increase in the order of polynomial completeness decreases the number of non-physical locking modes, but the decrease in the number of non-physical locking modes is slower than in finite elements.

In [29], Dolbow and Belytschko proposed a mixed formulation of the EFG method in combination with a selective reduced integration to reduce locking effects. They performed a numerical inf-sup test and showed that their method does not fulfill the LBB conditions which are necessary conditions for the stability and convergence of a mixed formulation. However, they pointed out similarities of the mixed EFG-method with four-node quadrilateral elements with constant pressure field which do not fulfill the LBB conditions but are stable for most applications. No pressure instabilities were observed for the wide range of problems they studied. Other approaches to avoid locking in meshfree methods were proposed e.g. by Wang and Chen [82], Chen et al. 
[26], Vidal et al. [81] and Huerta et al. [43].

\subsection{Approximation of the pressure field}

To avoid locking we will follow an idea by Hughes [44]. Other methods to avoid locking in meshfree methods have been proposed by Dolbow and Belytschko [29] and Chen et al. [26]. The deformation gradient is decomposed into a deviatoric and volumetric part, see Flory [34]:

$$
\mathbf{F}=\mathbf{F}^{\text {dev }} \mathbf{F}^{v o l}
$$

where the superimposed dev and vol indicates the deviatoric and volumetric part. In order to avoid locking, we will use a B-Bar technique where the deviatoric part will be approximated with higher-order shape functions as compared to the volumetric part. Therefore, $\mathbf{F}$ and $\overline{\mathbf{F}}$ are approximated by

$$
\begin{aligned}
\mathbf{F} & =\sum_{I \in \mathcal{N}} \nabla \Phi_{I}(\mathbf{X}) \mathbf{u}_{I}(t)+\sum_{I \in \mathcal{N}_{c}} \nabla \Phi_{I}(\mathbf{X}) S\left(f_{I}(\mathbf{X})\right) \mathbf{q}_{I}(t) \\
& +\underbrace{\sum_{I \in \mathcal{N}_{c}} \nabla S\left(f_{I}(\mathbf{X})\right) \Phi_{I}(\mathbf{X}) \mathbf{q}_{I}(t)}_{=0}+\mathbf{I} \\
\overline{\mathbf{F}} & =\sum_{I \in \mathcal{N}} \nabla \bar{\Phi}_{I}(\mathbf{X}) \mathbf{u}_{I}(t)+\sum_{I \in \mathcal{N}_{c}} \nabla \bar{\Phi}_{I}(\mathbf{X}) S\left(f_{I}(\mathbf{X})\right) \mathbf{q}_{I}(t) \\
& +\underbrace{\sum_{I \in \mathcal{N}_{c}} \nabla S\left(f_{I}(\mathbf{X})\right) \bar{\Phi}_{I}(\mathbf{X}) \mathbf{q}_{I}(t)}_{=0}+\mathbf{I}
\end{aligned}
$$

where the third term on the RHS of eq. (45) vanishes since the shear band is considered as open set. $\Phi_{I}(\mathbf{X})$ are shape functions based on a bilinear basis and are used in order to determine $\mathbf{F}^{\text {dev }}$ while the shape functions $\bar{\Phi}_{I}(\mathbf{X})$ use a constant basis and are used for the volumetric part of the B-Bar approach. The volumetric part of the deformation gradient is then

$$
\overline{\mathbf{F}}^{\text {vol }}=\frac{1}{3} \operatorname{tr}(\overline{\mathbf{F}}) \mathbf{I}
$$

with the associated pressure $\bar{p}$ is

$$
\bar{p}=K(\theta-1)
$$

with

$$
\theta=\operatorname{det}(\overline{\mathbf{F}})
$$


The deviatoric deformation gradient is computed by

$$
\mathbf{F}^{d e v}=\mathbf{F}\left(\mathbf{F}^{v o l}\right)^{-1}
$$

In finite elements, $q 1-p 0$ approaches tend to lead to instabilities. These instabilities can be eliminated by a stabilization procedure such as GLS or SUPG. The instabilities can be also prevented by using higher order shape functions. In our approach, we haven't observed any instabilities for the problems we tested but it would be straightforward to increase the polynomial basis.

\subsection{B-Bar formulation}

Substituting the approximations of the test and trial functions developed in section 2 and invoking the arbitrariness of $\delta \mathbf{u}_{I}$ and $\delta \mathbf{q}_{I}$ into eq. (8) we obtain

$$
\begin{array}{r}
\sum_{I \in \mathcal{N}} \delta \mathbf{u}_{I} \cdot\left(\mathbf{f}_{I}^{i n t}+\mathbf{M}_{I K}^{u u} \cdot \ddot{\mathbf{u}}_{K}+\mathbf{M}_{I K}^{u q_{1}} \ddot{\mathbf{q}}_{K}-\mathbf{f}_{I}^{e x t}\right)+ \\
\sum_{I \in \mathcal{N}_{c}} \delta \mathbf{q}_{I}\left(\mathbf{Q}_{I}^{i n t}+\mathbf{M}_{I K}^{u q_{1}} \cdot \ddot{\mathbf{u}}_{K}+\mathbf{M}_{I K}^{q q} \ddot{\mathbf{q}}_{K}-\mathbf{Q}_{I}^{e x t}+\mathbf{P}_{P}\right)=0
\end{array}
$$

where

$$
\begin{aligned}
\mathbf{f}_{I}^{\text {int }} & =\int_{\Omega \backslash \Gamma^{c}} \nabla \Phi_{I}(\mathbf{X}) \cdot \operatorname{dev}(\boldsymbol{\sigma}(\mathbf{X})) d \Omega+\int_{\Omega \backslash \Gamma^{c}} \nabla \bar{\Phi}_{I}(\mathbf{X}) \cdot \mathbf{I} \bar{p}(\mathbf{X}) d \Omega \\
\mathbf{f}_{I}^{e x t} & =\int_{\Omega \backslash \Gamma^{c}} \varrho \mathbf{b} \Phi_{I}(\mathbf{X}) d \Omega^{+} \int_{\Gamma^{t}} \overline{\mathbf{t}} \Phi_{I}(\mathbf{X}) d \Gamma \\
\mathbf{Q}_{I}^{i n t} & =\int_{\Omega \backslash \Gamma^{c}}\left(S\left(f_{I}(\mathbf{X})\right) \nabla \Phi_{I}(\mathbf{X})\right)^{S} \cdot \operatorname{dev}(\boldsymbol{\sigma}(\mathbf{X})) d \Omega \\
& +\underbrace{\int_{\Omega \backslash \Gamma^{c}} \Phi_{I}(\mathbf{X}) \nabla S\left(f_{I}(\mathbf{X})\right)^{S} \cdot \operatorname{dev}(\boldsymbol{\sigma}(\mathbf{X})) d \Omega}_{=0} \\
& +\int_{\Omega \backslash \Gamma^{c}}\left(S\left(f_{I}(\mathbf{X})\right) \nabla \bar{\Phi}_{I}(\mathbf{X})\right)^{S} \cdot \mathbf{I} \bar{p}(\mathbf{X}) d \Omega \\
& +\underbrace{\int_{\Omega \backslash \Gamma^{c}} \bar{\Phi}_{I}(\mathbf{X}) \nabla S\left(f_{I}(\mathbf{X})\right)^{S} \cdot \mathbf{I} \bar{p}(\mathbf{X}) d \Omega}_{=0}
\end{aligned}
$$




$$
\begin{aligned}
\mathbf{Q}_{\alpha I}^{e x t} & =\int_{\Omega \backslash \Gamma^{c}} \varrho \mathbf{b} S\left(f_{I}(\mathbf{X})\right) \Phi_{I}(\mathbf{X}) d \Omega^{+} \int_{\Gamma^{t}} \overline{\mathbf{t}} S\left(f_{I}(\mathbf{X})\right) \Phi_{I}(\mathbf{X}) d \Gamma \\
& -\left(\int_{\Gamma^{c}} \mathbf{t}_{c} \Phi_{I}(\mathbf{X}) \llbracket S\left(f_{I}(\mathbf{X})\right) \rrbracket+\beta \Phi_{I}(\mathbf{X}) \llbracket S\left(f_{I}(\mathbf{X})\right) \rrbracket \dot{\mathbf{u}}\right) d \Gamma \\
\mathbf{P}_{P} & =\int_{\Gamma^{c}} \alpha \Phi_{I}(\mathbf{X}) \Phi_{I}(\mathbf{X}) \llbracket \mathbf{u}_{n} \rrbracket d \Gamma
\end{aligned}
$$

where we have added a visous term, last term in equation (54), in order to avoid oscillations. The mass matrix is

$$
\mathbf{M}_{I J}=\left[\begin{array}{ll}
\mathbf{m}_{I J}^{u u} & \mathbf{m}_{I J}^{u q} \\
\mathbf{m}_{I J}^{q u} & \mathbf{m}_{I J}^{q q}
\end{array}\right]
$$

with

$$
\begin{aligned}
& \mathbf{m}_{I J}^{u u}=\int_{\Omega \backslash \Gamma^{c}} \varrho_{0} \Phi_{I}(\mathbf{X}) \hat{\Phi}_{J}(\mathbf{X}) \mathbf{I} d \Omega \\
& \mathbf{m}_{I J}^{u q}=\int_{\Omega \backslash \Gamma^{c}} \varrho_{0} \Phi_{I}(\mathbf{X}) \Phi_{J}(\mathbf{X}) S\left(f_{J}(\mathbf{X})\right) d \Omega \\
& \mathbf{m}_{I J}^{q q}=\int_{\Omega \backslash \Gamma^{c}} \varrho_{0} \Phi_{I}(\mathbf{X}) S\left(f_{I}(\mathbf{X})\right) \Phi_{J}(\mathbf{X}) S\left(f_{J}(\mathbf{X})\right) d \Omega
\end{aligned}
$$

A selective reduced integration is chosen. For the part of the nodal internal forces that depends on the pressure, the second term in eqs. (51) and (53), we employed a nodal integration. Stress point integration is used for the deviatoric terms. A stress point is arranged in the middle of each cell (in 2D) or cuboid (in 3D) spanned by four and eight particles, respectively; see Rabczuk and Belytschko [72], Rabczuk et al. [75] for our construction of stress points. One point quadrature was used for the surface integrals $\Gamma_{I}^{c}$.

We used a second-order explicit central difference time integration scheme.

\subsection{H-Adaptivity}

We use a gradient based error estimator. In other words, particles are added where large strain gradients (multiplied with the mesh density) occur. Due to computational cost we allow depending on the problem between 2 to 5 refinement steps. The adaptive refinement strategy for structured particle discretizations is extensively explained in Rabczuk and Belytschko [74] and the 
error estimation in Rabczuk and Belytschko [73] and will not be described within this manuscript.

\section{EXAMPLES}

\subsection{Plate under compression}

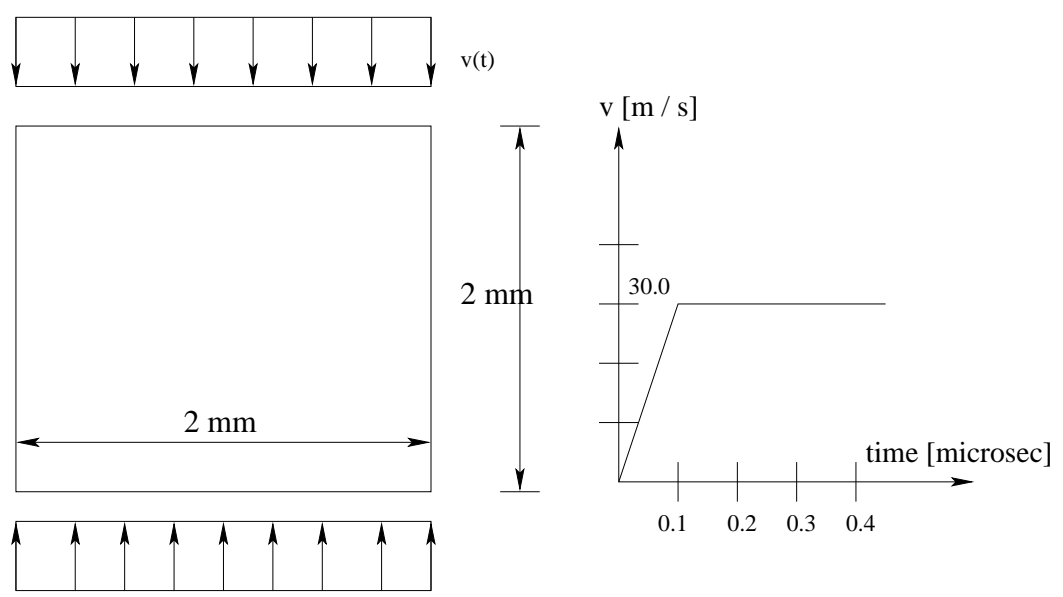

Fig. 2. Model description for the quadratic plate under compression

Consider a specimen under compression as shown in figure 2 with an imperfection at $x_{0}=0.0, y_{0}=0.0$. This example was studied by Needleman [62], Lemonds and Needleman [52] and Belytschko et al. [14] with a viscoplastic constitutive model. In [14], the imperfection is realized by modifying the yield strength:

$$
\bar{\sigma}_{y}(x, y)=\sigma_{y}\left(1-\alpha e^{-\left(x-x_{0}\right)^{2}-\left(y-y_{0}\right)^{2} / r_{0}^{2}}\right)
$$

with $\alpha=0.2$ or

$$
\begin{aligned}
& \bar{\sigma}_{y}(x, y)=\sigma_{y}\left(0.9+0.01\left[\left(x-x_{0}\right)^{2}-\left(y-y_{0}\right)^{2}\right] / r_{0}^{2}\right) \quad r<r_{0} \\
& \bar{\sigma}_{y}(x, y)=\sigma_{y} \quad r \geq r_{0}
\end{aligned}
$$

Belytschko et al. [14] also presented results for different size of the imperfection $r_{0}$. They showed that with smaller imperfection, multiple shear banding occurs. We have chosen an imperfection of $r_{0}=0.1 \mathrm{~mm}$ and used the $J_{2}$-plasticity model from section 4.1.1.

We used this example to test our approach. No adaptivity was used. Figure 3 shows the shear band with the effective plastic strains at different time steps 
for approximately 40,000 particles. The shear band agrees very well with the shear band obtained in Lemonds and Needleman [52], Needleman [62] and Belytschko et al. [14].

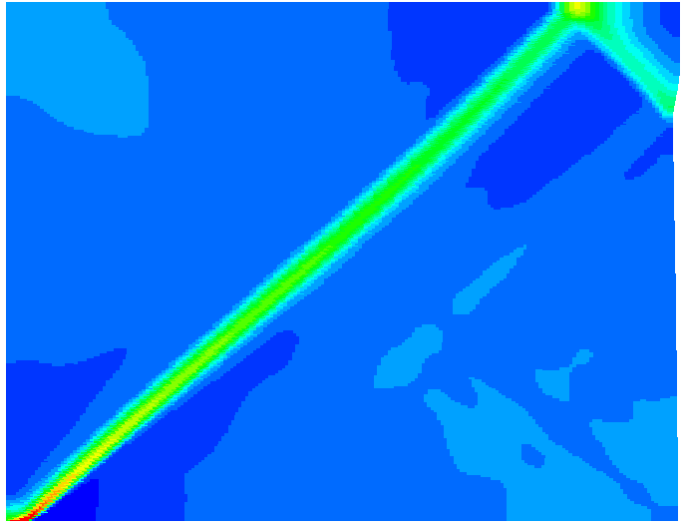

a) $0.0038 \mathrm{~ms}$

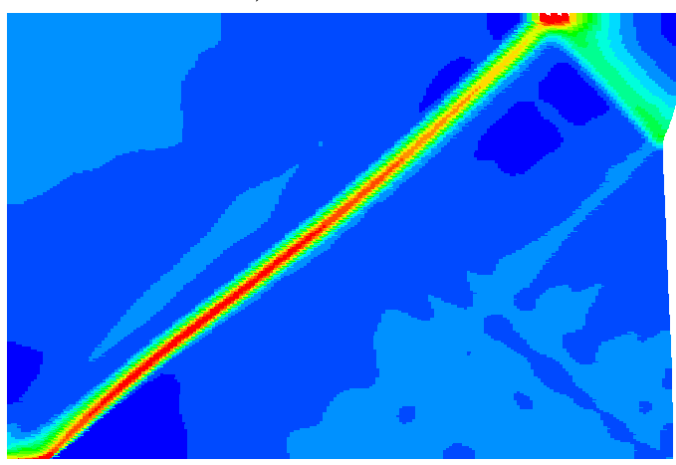

c) $0.006 \mathrm{~ms}$

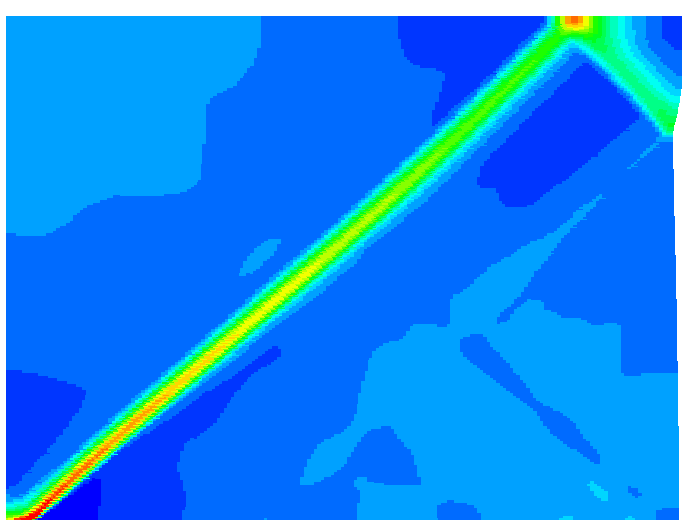

b) $0.0042 \mathrm{~ms}$

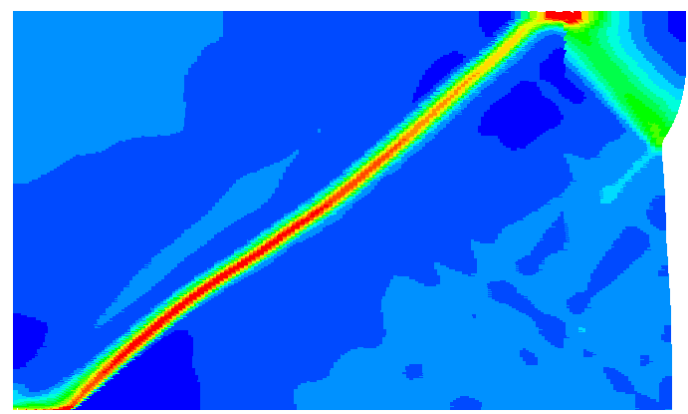

d) $0.00725 \mathrm{~ms}$

Fig. 3. Effective plastic strain in the plate under compression at different time steps

\subsection{Plate with two holes}

The next example is a plate with two holes that is loaded in compression as shown in figure 4. Diez et al. [28] studied this example using a viscoplastic constitutive model. The load was applied as velocity boundary condition with constant velocity of $1 \mathrm{~m} / \mathrm{s}$. They modified the location of the holes and found different shear band pattern. We study this example with the same constitutive model as described in section 4.3. We started initially with 480 particles and 750 particles. We allowed up to 5 and 4 refinement steps that resulted in almost 2900 and 3600 particles, respectively. The material parameters are according to Diez et al. [28] Young's modulus $E=200 G P a$, Poisson's ratio $\nu=0.3$, initial yield strength $\sigma_{0}=200 M P a$, softening modulus $H=-20 G P a, \gamma=500 s^{-1}$ and $N=1$.

The shear band at the end of the simulation is shown in figure 5 for the two 


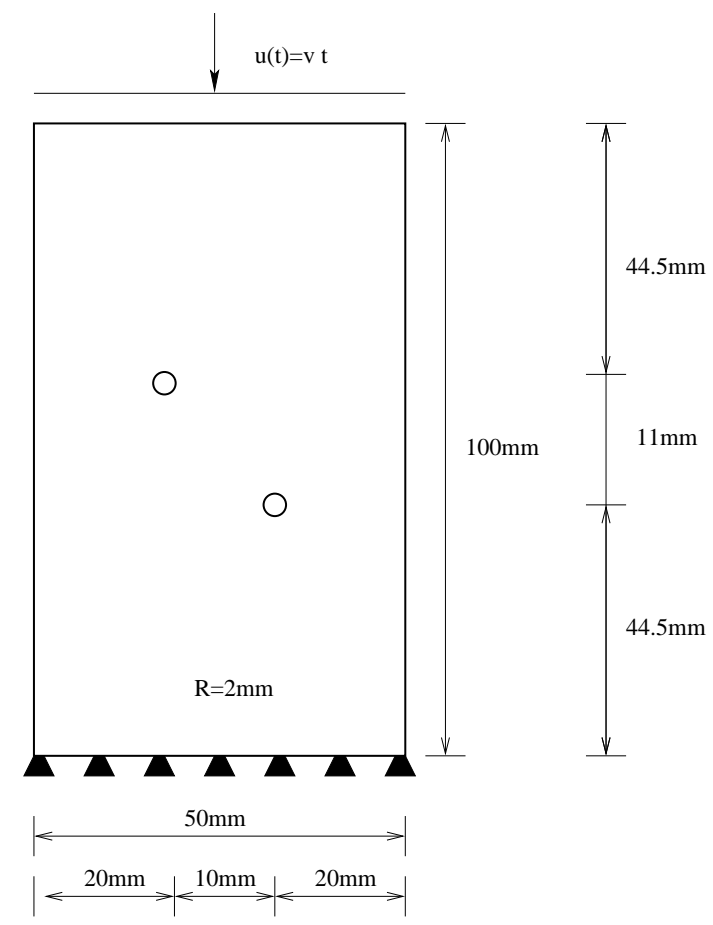

Fig. 4. Model description for the plate with two holes under compression

particle discretization. The results agree well with the results of Diez et al. [28]. The load displacement curve is shown in figure 6 and is very similar to the one in [28].

\subsection{Nesterenko problem}

Meyers et al. [57], Nesternko et al. [64], Shih et al. [78], Xue et al. [87] performed a series of multiple shear banding experiments in cylinders for different type of materials and different dimensions. The test-set up is shown in figure 7. In all experiments, the cylindrical test specimen is surrounded by a copper stopper tube and a copper driver tube, that is itself surrounded by an explosive. We will consider here the stainless steel specimen. We carried out three dimensional computations and modelled also the explosive. We use the Johnson Cook model for copper. We also assume that the plastic work is completely transformed into heat. The material data are density $\varrho=8.92 \mathrm{~g} / \mathrm{cm}^{3}$, bulk modulus $K=115 G P a$, shear modulus $G=44 G P a$, melting temperature $T_{m}=1058 \mathrm{~K}, A=90 \mathrm{MPa}, B=292 \mathrm{MPa}, n=0.31, C=0.025, m=1.09$ and specific heat $c_{v}=385 \mathrm{~J} / \mathrm{kg} \mathrm{K}$. For stainless steel, the material parameters are density $\varrho=7.9 \mathrm{~g} / \mathrm{cm}^{3}$, bulk modulus $K=200 G P a$, shear modulus $G=$ 


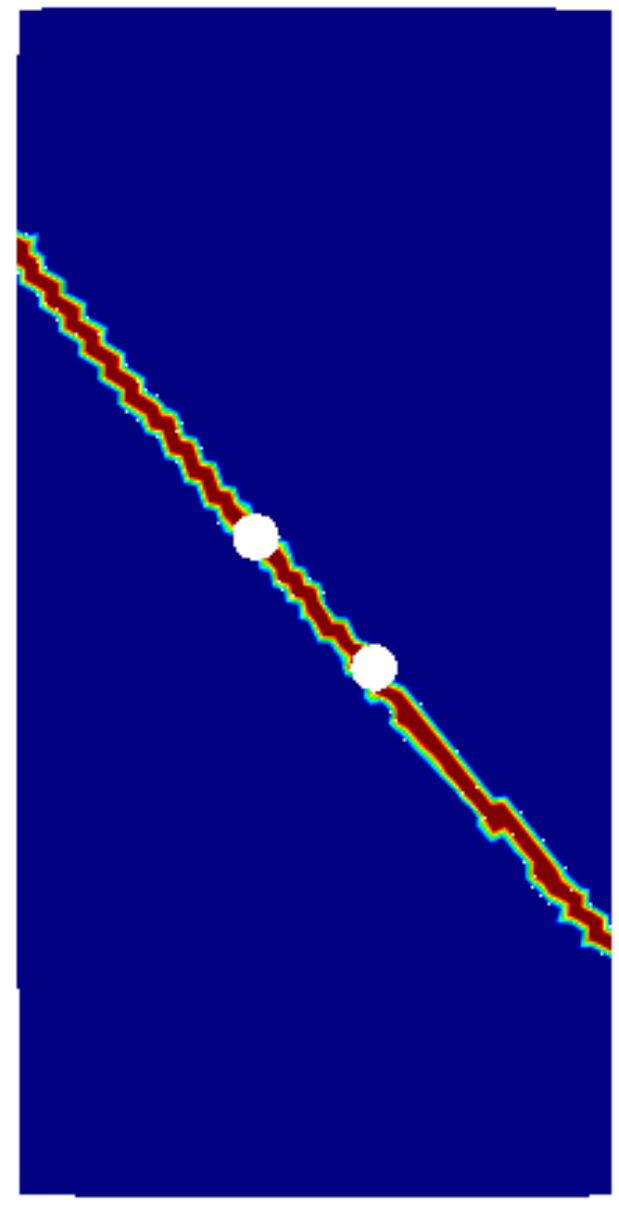

a) 480 particles

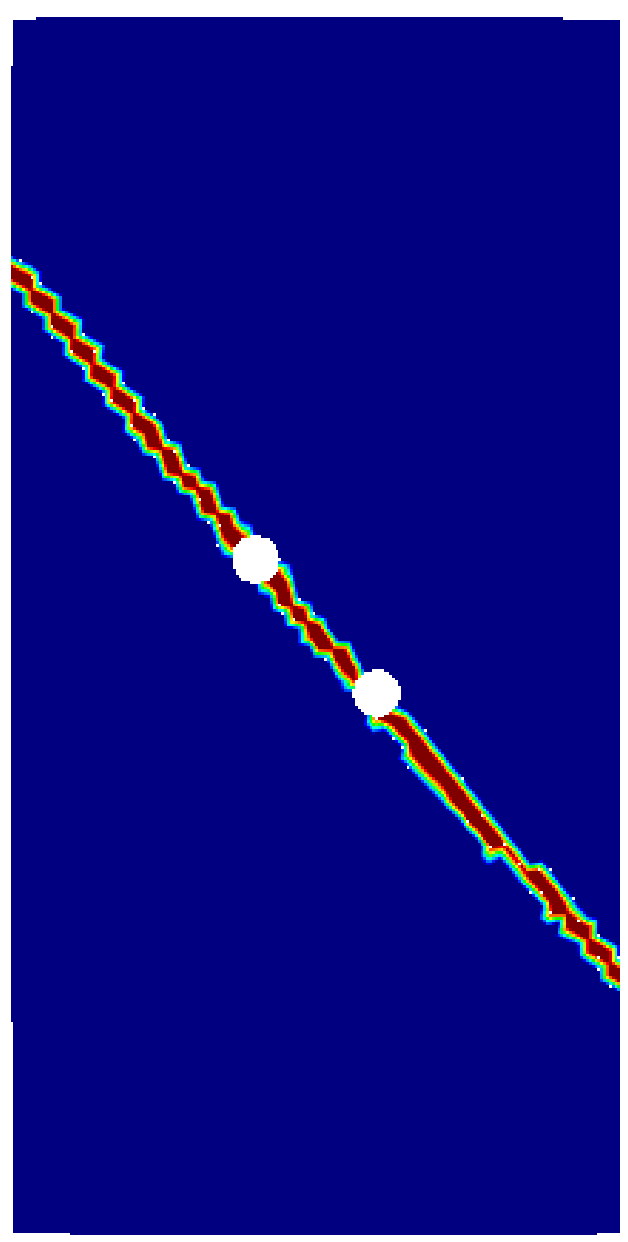

b) 750 particles

Fig. 5. a),b) Shear band pattern of the two-hole-problem

77.5 GPa, melting temperature $T_{m}=1396 \mathrm{~K}, A=110 \mathrm{MPa}, B=1500 \mathrm{MPa}$, $n=0.36, C=0.014, m=1$ and specific heat $c_{v}=500 \mathrm{~J} / \mathrm{kg} \mathrm{K}$.

Figure 8 shows the shear band pattern at the end of the computation for approximately 130,000 particles. The shear band does not pass through the entire specimen what corresponds well with the experimental observations. Figure 9 shows shear band patterns at four different "levels" of the specimen, from the top up to the middle. The shear band pattern is almost symmetric over the length. As can be seen, the shear band crosses over the entire length of the cylinder. The crack front is slightly curved and advances most in the middle of the cylinder, figure $9 \mathrm{~d}$. 


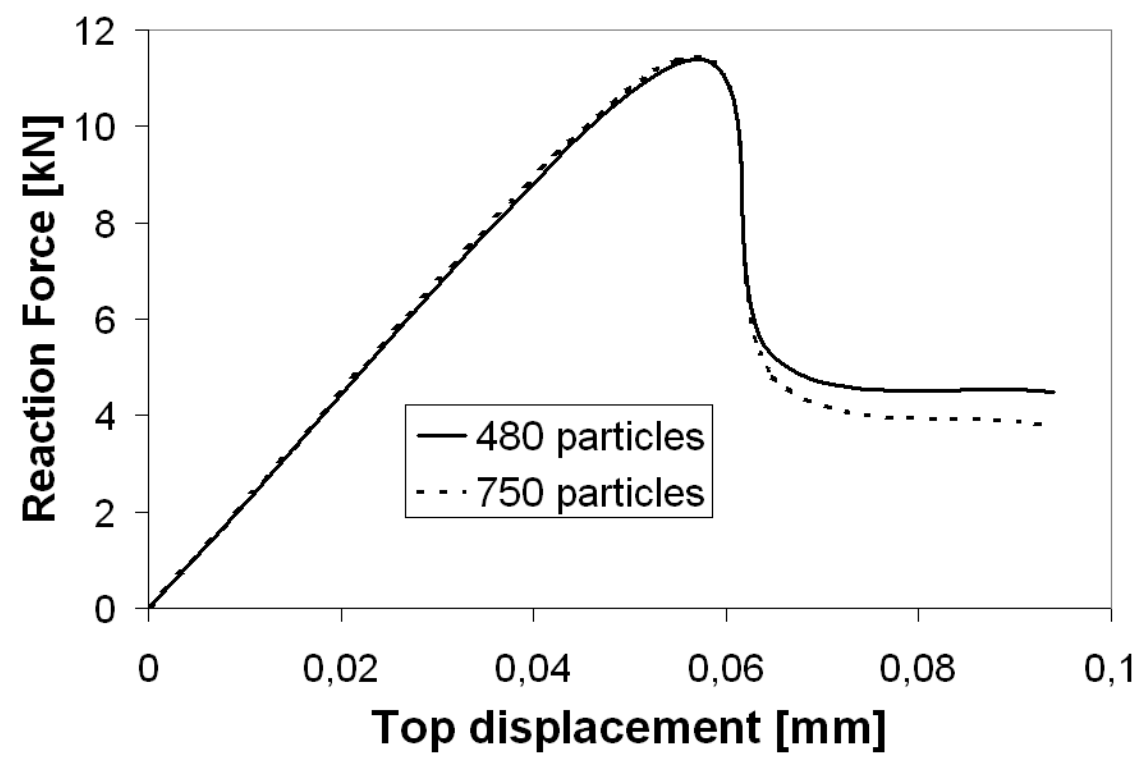

Fig. 6. Load displacement curve of the two-hole problem

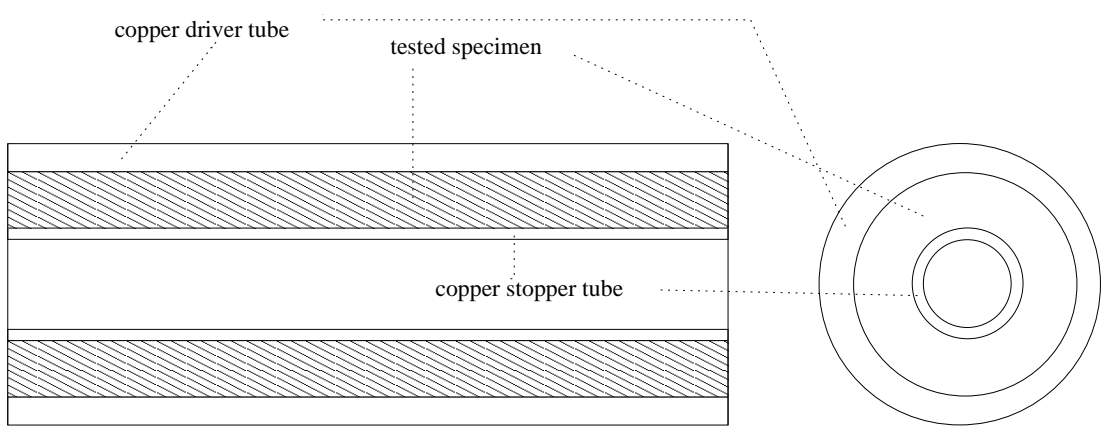

Fig. 7. Typical test-setup of the Nesternko problem

\subsection{Double notched block under tension}

Consider a specimen of dimensions $L \times W \times T$ with two notches of the length $a$ as shown in figure 10. The specimen is subjected to uniaxial tensile loading. This example was studied by Li et al. [53] using meshfree method and Ewing and Hill [32] analytically. We used the constitutive model in section 4.1.1 and studied different refinements from 18,000 particles up to approximately 125,000 particles. Note that we did not apply the adaptive refinement scheme in this example. We did this for better comparison to the results in [51] who did not use adaptive refinement. The final shear band pattern for these two discretization is shown in figure 11. While the shear band pattern of Lee et al. [51] differs from the solution of Ewing and Hill [32], our results agree very well with the solution of Ewing and Hill [32] even for very coarse particle discretization. 


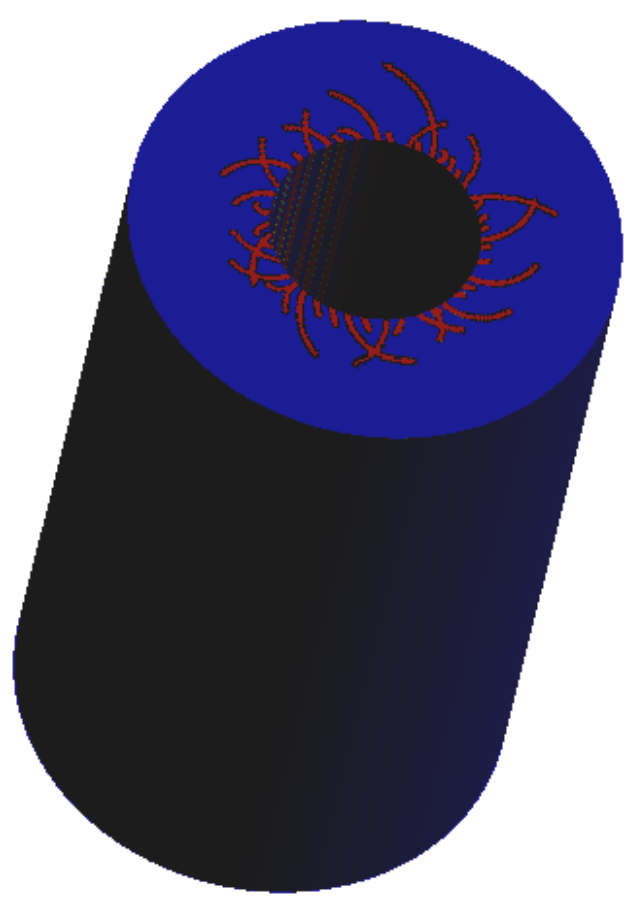

a)

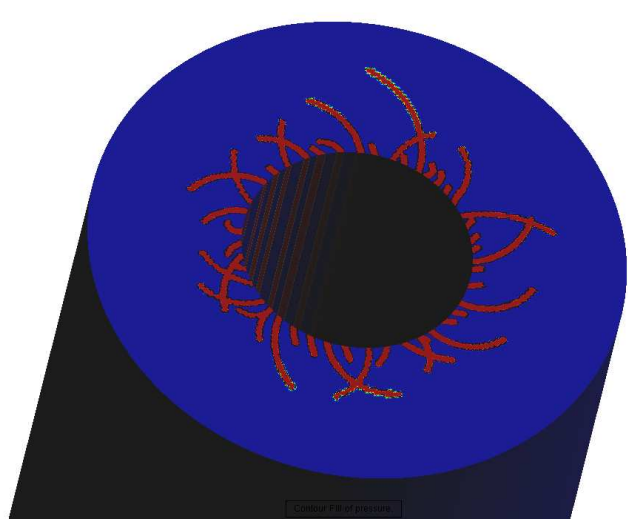

b)

Fig. 8. Shear band pattern for the Nesterenko problem

\subsection{Cutting of a block}

Consider cutting out of a block. The cutter is modelled as rigid body and has an inclination of 80 degrees against the horizontal axis. It will be moved with constant speed in $\mathrm{x}$-direction and is fixed in $z$-direction and $y$-direction. The block is discretized with approximately 900 particles in the initial configuration where we have used a coarser discretization far away from the load. We allow up to 4 refinement steps that resulted in approximately 55,000 particles in the final configuration. This example shows the applicability of the method to extreme large deformation with fragmentation and contact. We use the plasticity model explained in section 4.1.1.

The effective plastic strain of the block at different time steps and different deformations states is shown in figure 12. For a better illustration, additional interpolation points are added. The deformed adaptive mesh is shown in figure 13. A cut-out in the three different planes ( $\mathrm{x}-\mathrm{y}$ plane, $\mathrm{x}-\mathrm{z}$ plane and $\mathrm{y}-\mathrm{z}$ plane) is shown in figure 14 and illustrates better the $3 \mathrm{D}$ refinement stages.

\subsection{The Kalthoff problem}

Kalthoff and Winkler [47] and Kalthoff [48] performed a series of experiments 


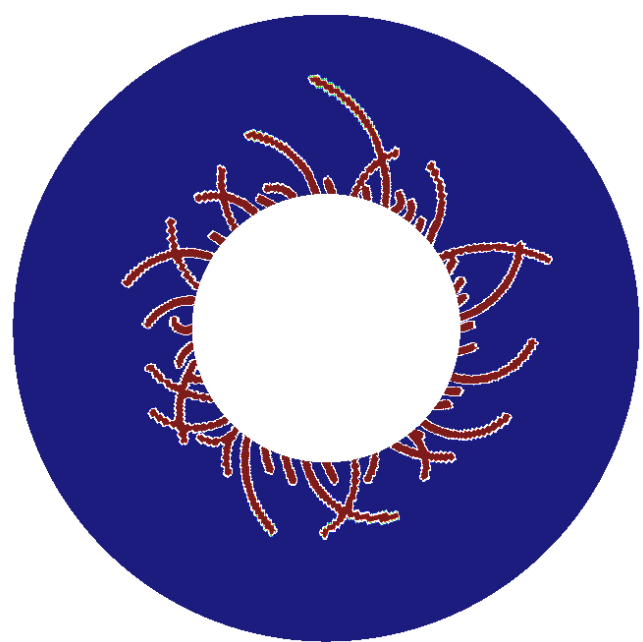

a) top $(70 \mathrm{~cm})$

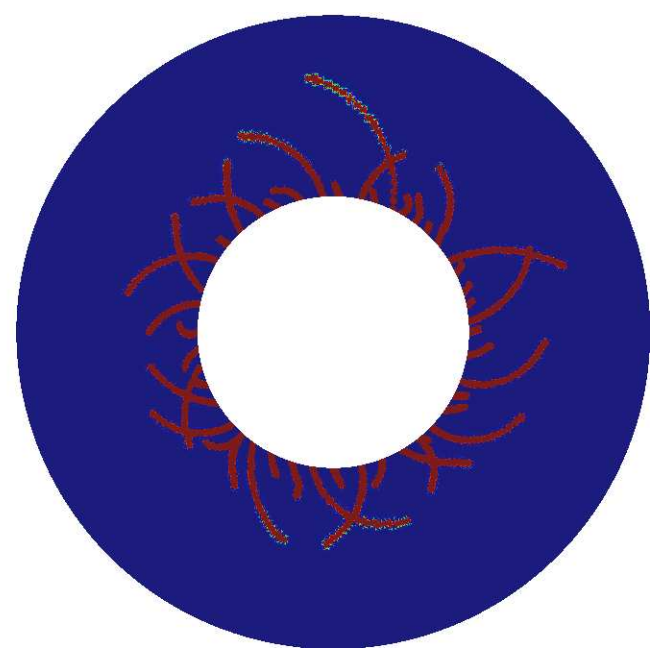

c) top $(48 \mathrm{~cm})$

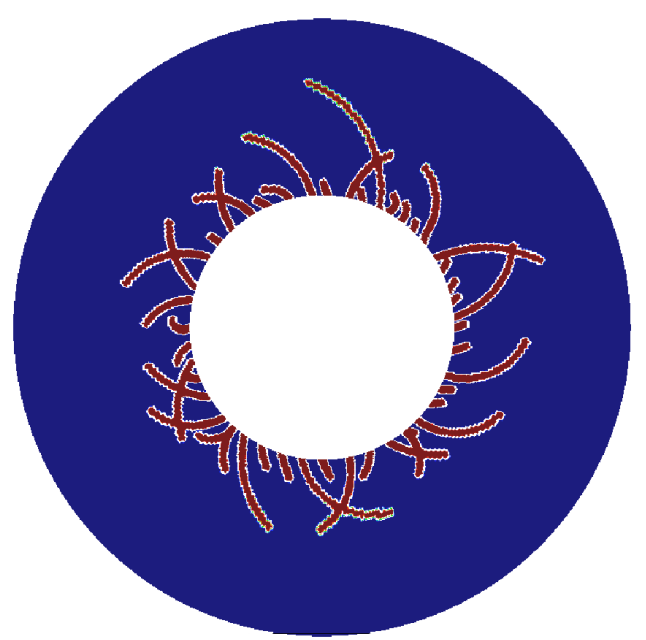

b) $60 \mathrm{~cm}$

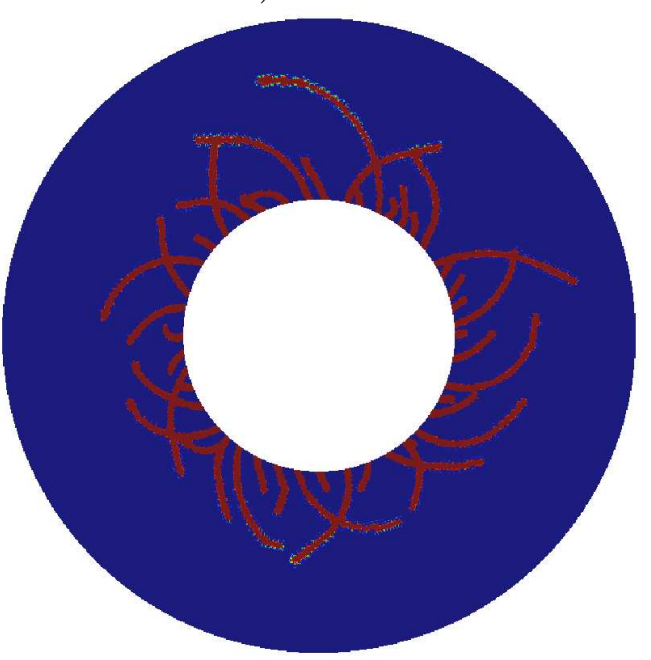

d) middle $(35 \mathrm{~cm})$

Fig. 9. Shear band formation for the Nesterenko problem at different levels measured from the top of the specimen; see also figure 7

where a steel plate is subjected to impact loading with different impact velocities as shown in figure 15. They discovered that the failure mode depends on the impact velocity. Up to a certain velocity of the impactor $v_{c}$, the steel plate undergoes brittle fracture: a crack develops at a 70 degree angle to the horizontal axis, see figure 16a. When exceeding $v_{c}$, they found a shear band developing from the notch, figure 16b. We will focus in our studies on experiments done with steel and sharp notches with small radius, so that we expect failure mode transition with $v_{c}=30 \mathrm{~m} / \mathrm{s}$.

Numerical studies of the Kalthoff and related experiments include Batra and Gummalla [6], Batra and Jaber [7], Li et al. [54], Needleman and Tvergaard [63], Zhou et al. [90]. Mostly two-dimensional computations under the assumption of plane strain conditions were performed. The impact was usually 


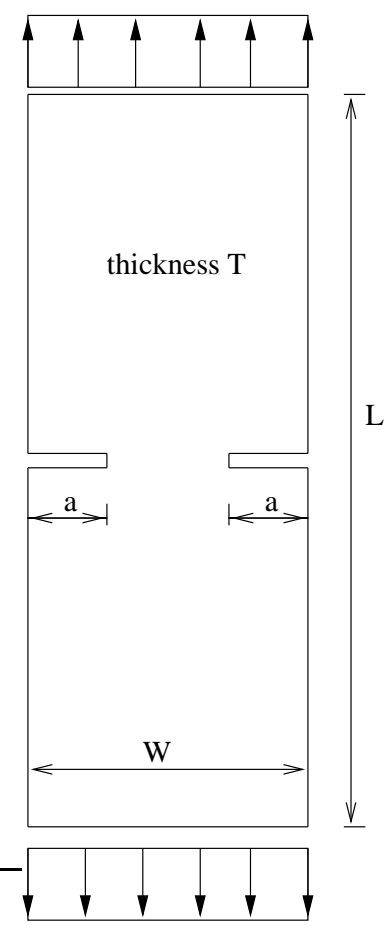

Fig. 10. Tensile specimen with two notches

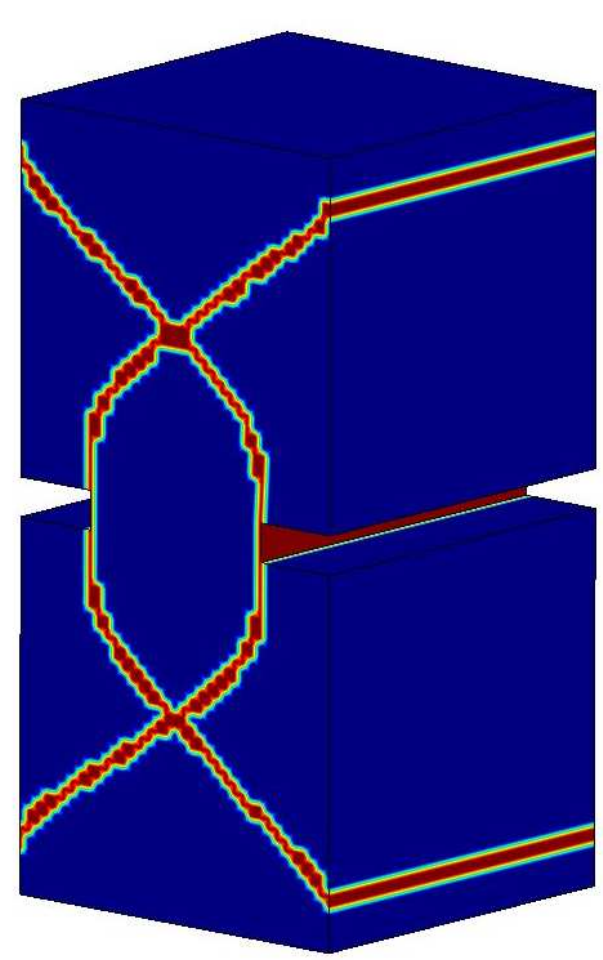

a) 18,000 particles

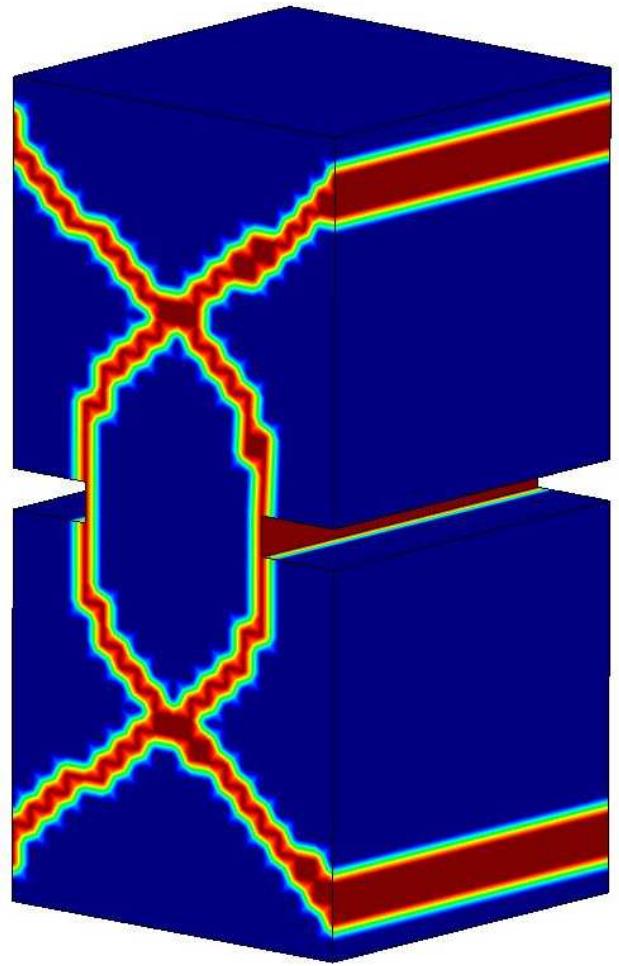

b) 125,000 particles

Fig. 11. Tensile specimen with two notches

modelled by a velocity boundary condition. Three-dimensional simulations have been done, e.g. by Batra and Ravisankar [9]. Batra et al. [8] studied the 


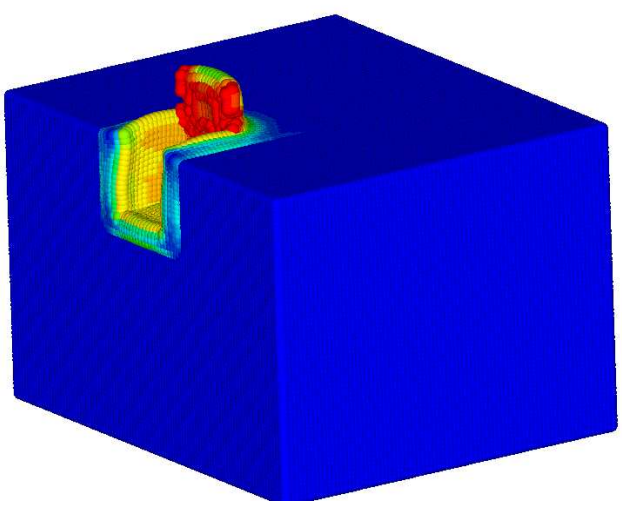

a)

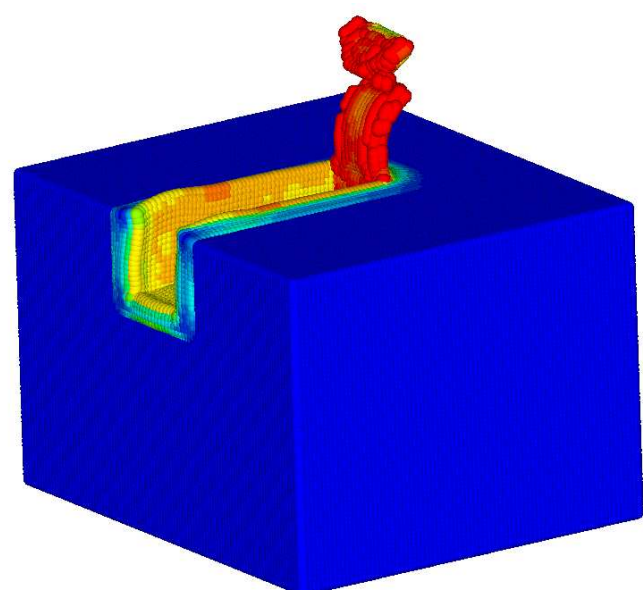

c)

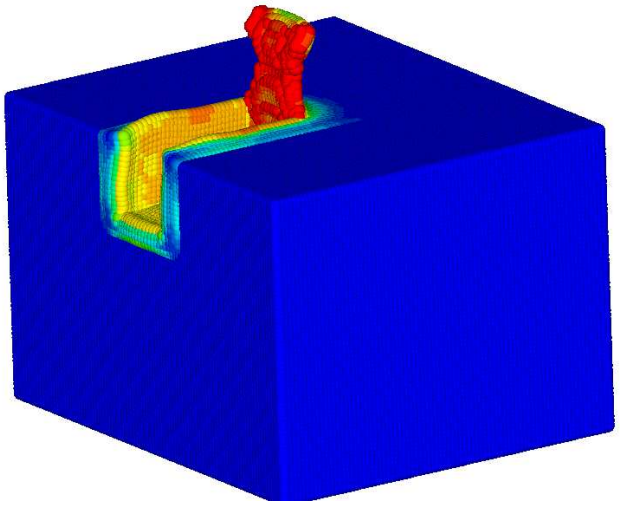

b)

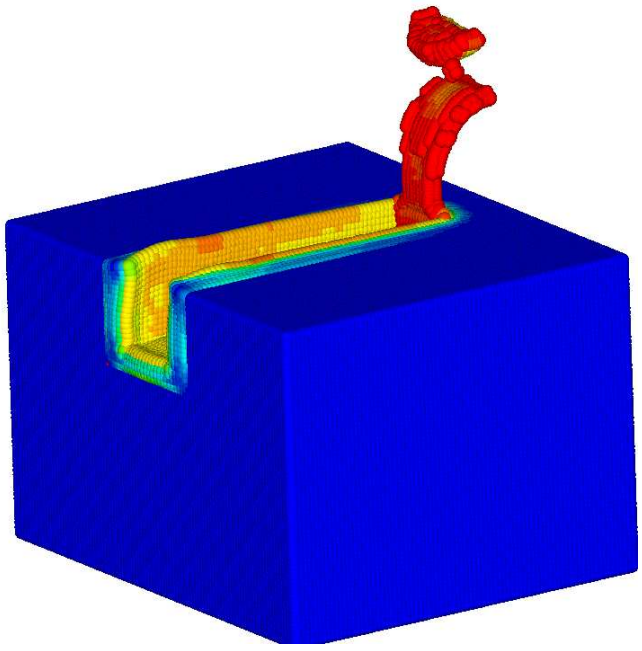

d)

Fig. 12. Cutting of a block at different time steps; the effective pastic strain is shown

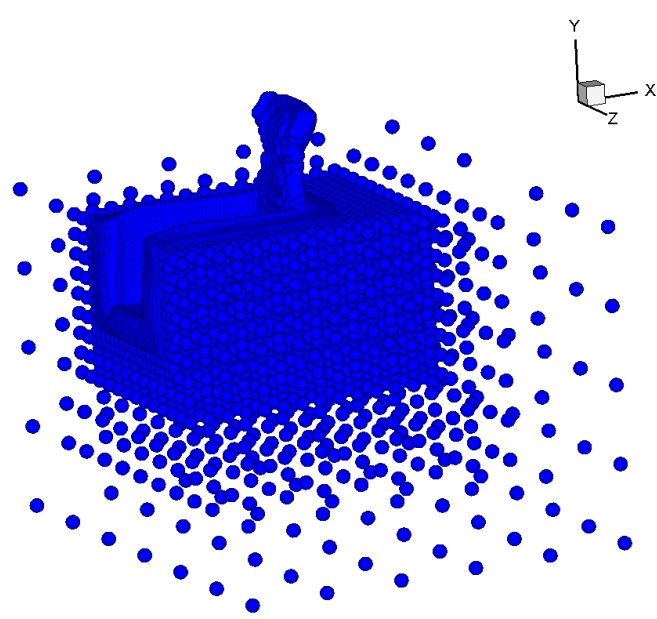

a)

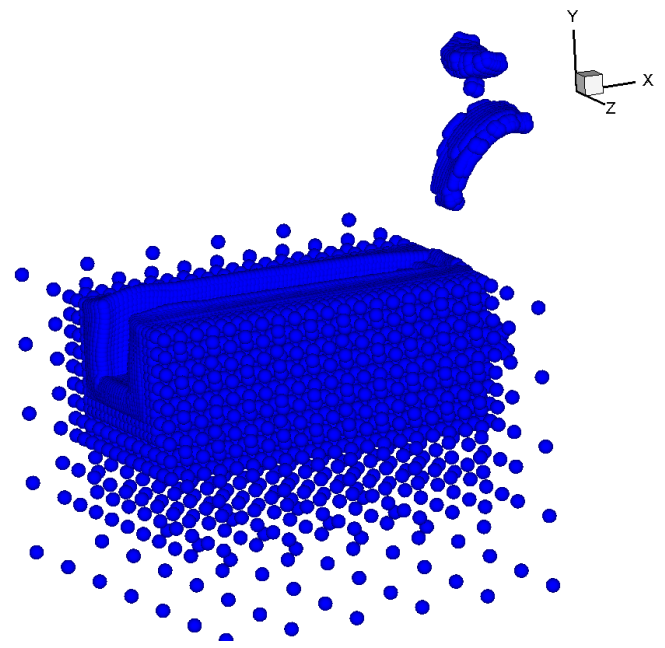

b)

Fig. 13. Cutting of a block at different two different time steps; adaptive refinement 


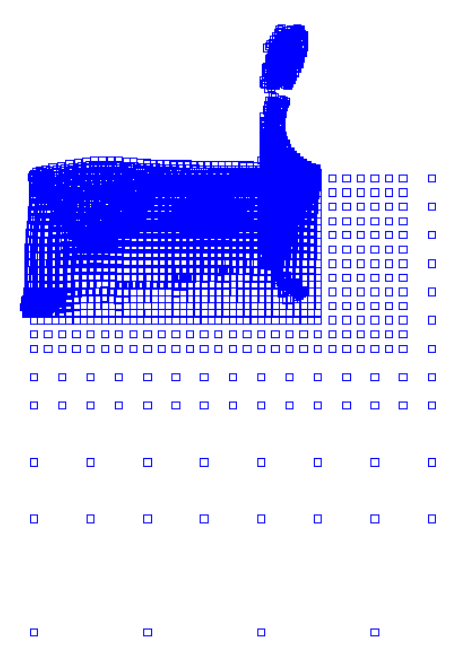

a) x-y plane

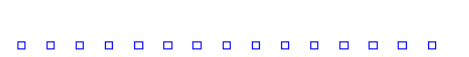

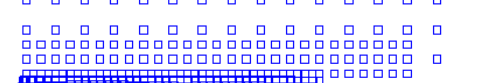

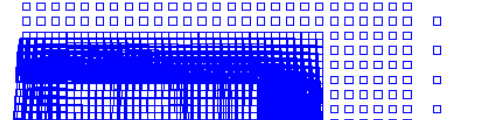

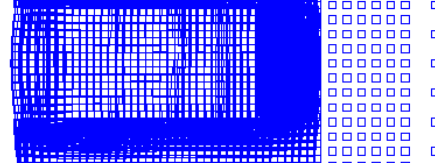

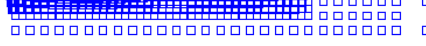

b) x-z plane

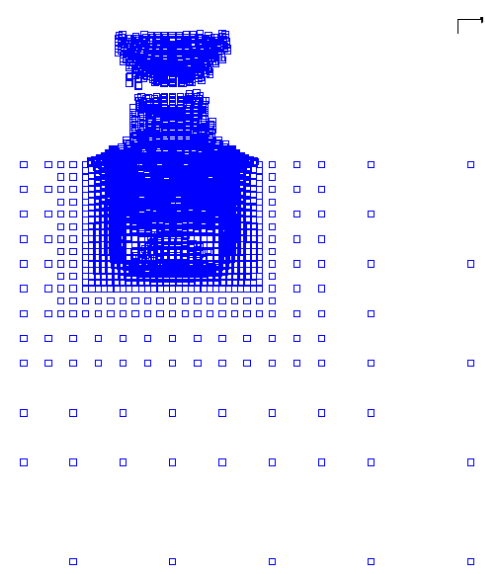

b) y-z plane

Fig. 14. 2D view of the cutting block

influence of the notch and reported that the shape of the notch plays an important role in determining the transition between brittle-to-ductile fracture. For example, for a sharp elliptic notch, they showed that ductile failure occurs at relatively low impact velocities (around $20 \mathrm{~m} / \mathrm{s}$ ) but for a circular notch, the transition from brittle-to-ductile fracture takes place at much higher impact velocities. We modelled the notch by enriching the nodes and setting the tractions to zero. This way, we eliminated the influence of the notch shape on the results that can be critical in a numerical simulation.

In our computations we used the Johnson-Cook model [46], section 4.2, with material parameters $A=792 M P a, B=509 M P a, C=0.014, n=0.26$, $m=0.55$. The density is $\varrho=7800 \mathrm{~kg} / \mathrm{m}^{3}, K=157 \mathrm{GPa}, \nu=76 \mathrm{GPa}$ and the 


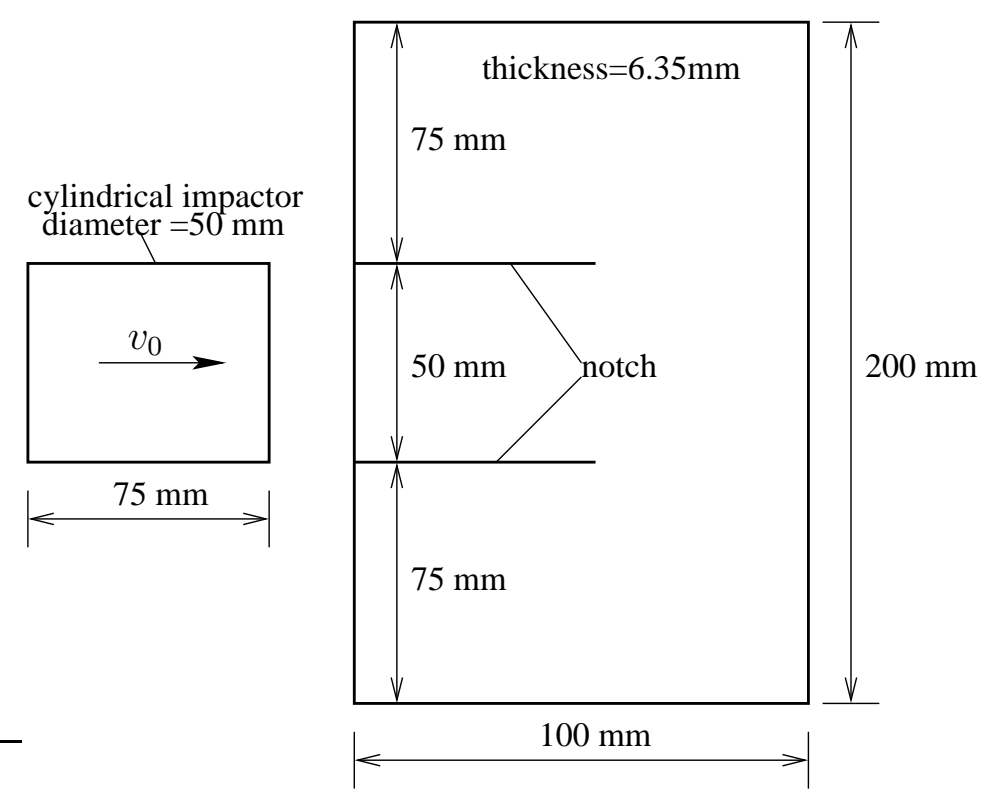

Fig. 15. The Kalthoff problem: test setup

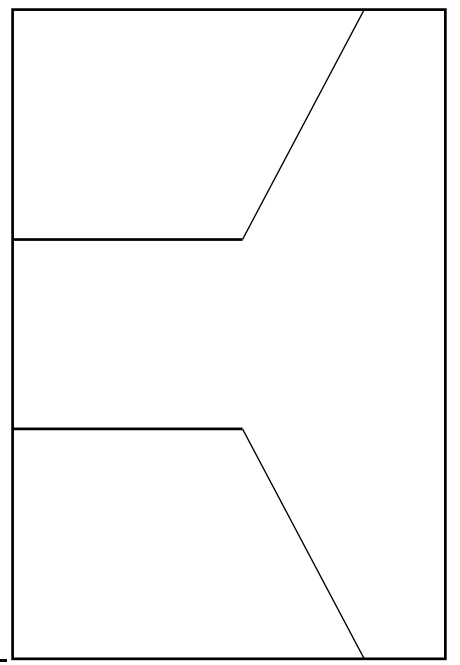

a)

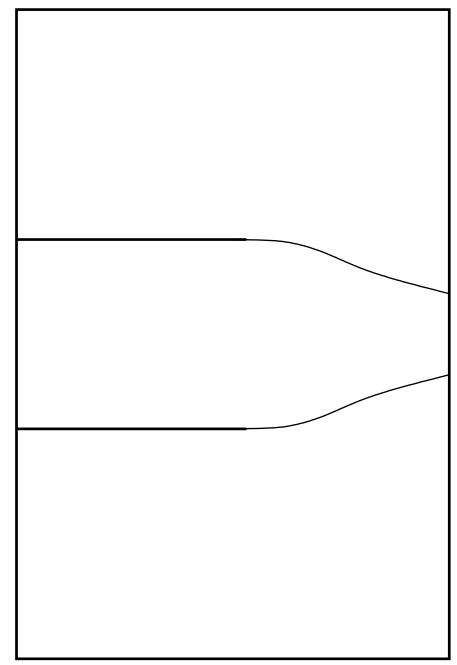

b)

Fig. 16. Different failure modes of the Kalthoff problem, a) brittle, b) ductile specific heat is $c_{v}=477 \mathrm{~J} / \mathrm{kgC}, T_{r}=296 \mathrm{~K}, T_{m}=1033 \mathrm{~K}$ and $\beta=1$. As was pointed out by Gummalla [38], the initial negative slope of the effective plastic stress-effective plastic strain curve highly determines the shear band initiation and the original failure surface is not able to capture this effect. He suggested the following form of the effective yield stress that gave better results in his computations:

$$
\sigma_{Y}=\max \left[\left(A+B \gamma^{n}\right)\left(1+C \ln \dot{\epsilon}^{*}\right)\left(1-\delta \exp \left(\left(T-T_{0}\right) / \kappa_{0}-1\right)\right), 0\right]
$$

with $A=2 G P a, B=94.5 G P a, C=0.0165, T_{0}=293 K, \gamma_{0}=1.310^{-} 13 s^{-1}$ 


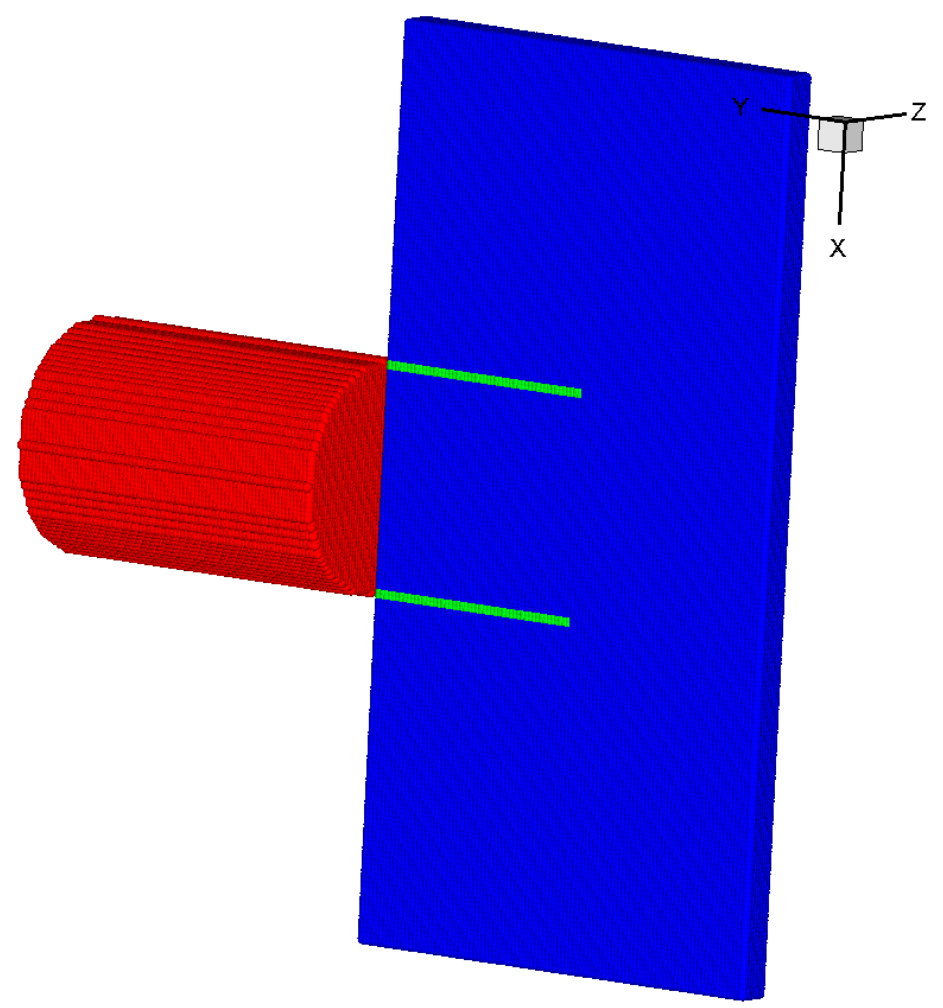

Fig. 17. Initial discretiaztion for the Kalthoff problem

and $\kappa=500 K$.

We tested three different particle arrangements. The first two particle discretizations had initially 18,000 particles and 110,000 particles and allowed a maximum of 3 and 2 refinement steps, respectively. In the last particle configuration, we used an unstructured particle discretization. The numbers of particles almost doubled for the course discretization while the numbers of particles increased to approximately 140,000 particles for the finer discretization. The impactor is also discretized as shown in figure 17.

First, we will present the results with an impact velocity of $40 \mathrm{~m} / \mathrm{s}$. We are able to capture the principal shear band pattern observed in the experiment independent from the mesh size. A two-dimensional view is presented in figure 18.

The final shear band pattern for an impact velocity of $20 \mathrm{~m} / \mathrm{s}$ is shown in figure 19. Note that the simulation is 3D though the results are plotted only in the $\mathrm{x}-\mathrm{y}$ plane. As in the experiment, we obtain a brittle failure where a crack propagates with an angle of approximately 70 degrees. 


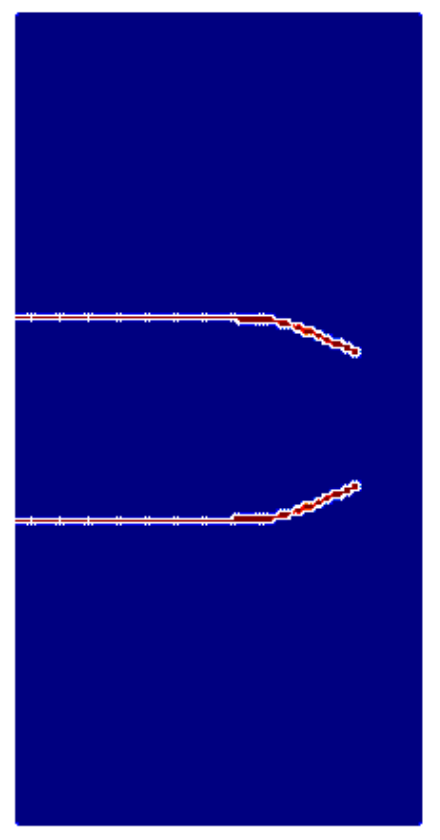

a) 35,000 particles

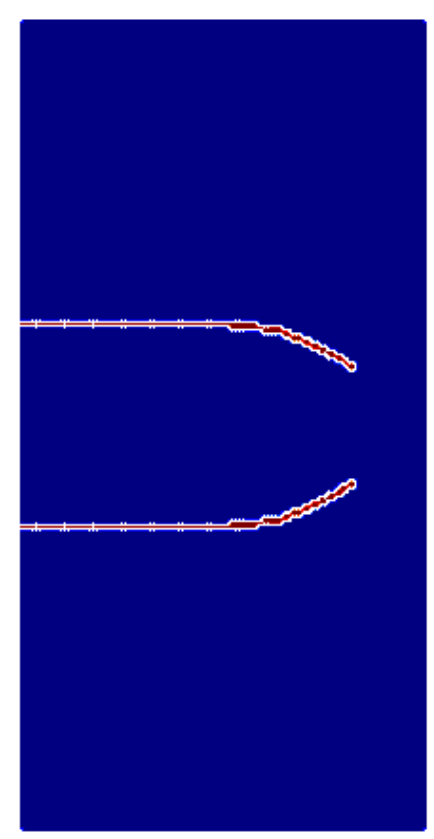

b) 250,000 particles

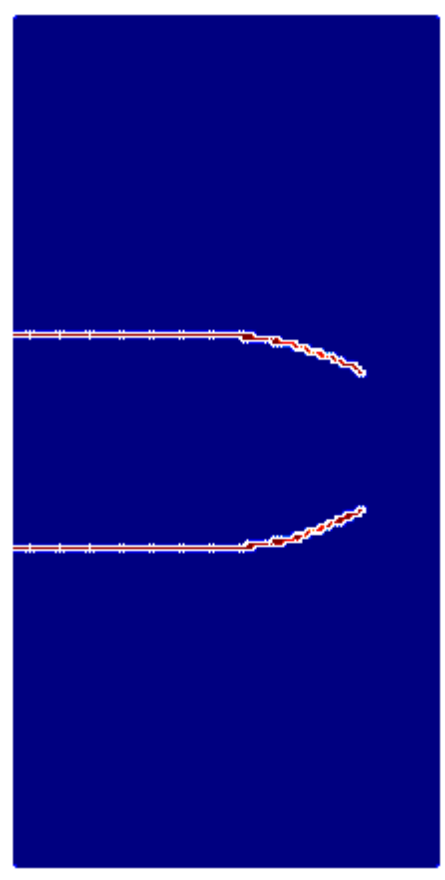

c) 30,000 particles

Fig. 18. Shear band pattern for the Kalthoff problem at impact velocity of $40 \mathrm{~m} / \mathrm{s}$ for different initial particle discretizations and a),b) structured meshes and c) unstructerd mesh; ductile failure occurs, 2D view

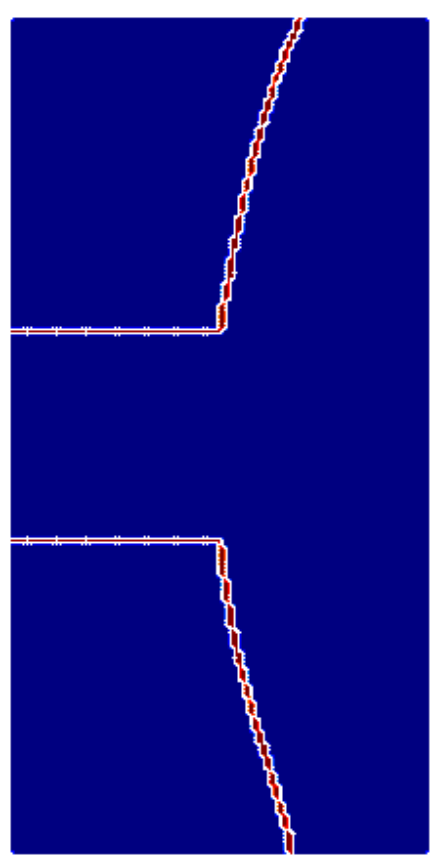

a) 35,000 particles

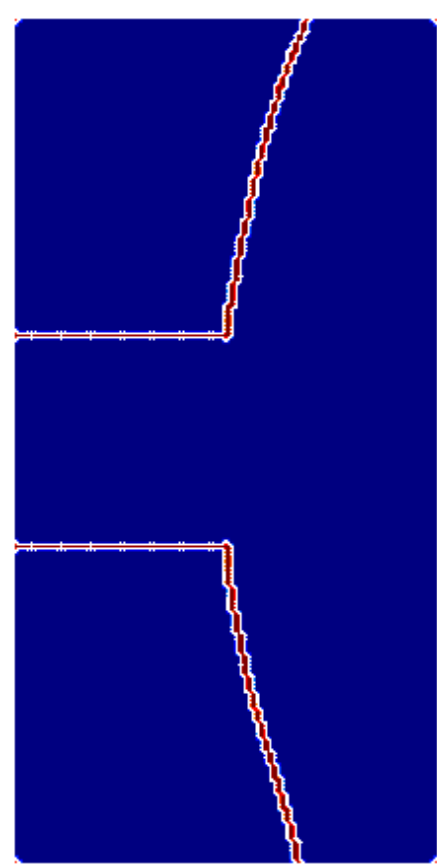

b) 250,000 particles

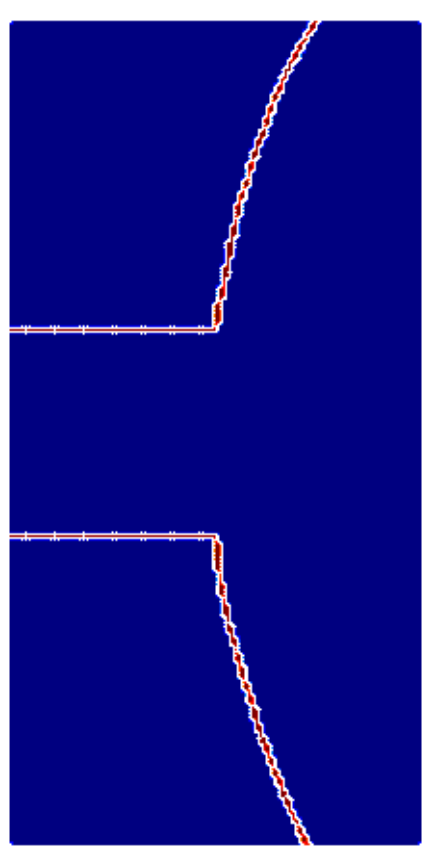

c) 30,000 particles

Fig. 19. Crack pattern for the Kalthoff problem at impact velocity of $20 \mathrm{~m} / \mathrm{s}$ for different initial particle discretizations and a),b) structured meshes and c) unstructerd mesh; brittle failure occurs 


\section{CONCLUSIONS}

We have presented an adaptive meshfree method for modelling shear bands with cohesive surfaces. Loss of material stability is chosen as transition criterion from continuum to discontinuum. The shear band is hereby represented as set of cracked segments located at the particle positions. To suppress opening of the discontinuity, we introduced a penalty. Otherwise, the cohesive law is completely described in terms of tangential jumps in the displacement or velocity, respectively. Although no remeshing is necessary, a certain refinement around the shear band tip is needed to obtain acceptable results. Adaptive refinement is ideally suited to improve accuracy only in a local region where needed. It is particularly simple to implement in a meshfree context and well suited for crack and shear band problems where high gradients and large plastic strains occur only in a very local or narrow region. Especially in three dimensions, non-adaptive methods soon find their limits due to lack of computer power as e.g. noticed by Gasser and Holzapfel [35] who modelled 3D crack propagation in unreinforced concrete.

Our method can naturally handle initiation and propagation of a single shear band as well as multiple shear banding with branching, intersection and contact. The method is based on a coupled Lagrangian/Eulerian kernel formulation. Initially, a Lagrangian kernel is employed that guarantees that shear bands occur physically and not due to numerical artifacts. After shear band initiation, an Eulerian kernel guarantees the stability of the method for extremely large deformation as demonstrated for a cutting example with fragmentation. In the blending region, particles across the shear band are excluded from the domain of influence. Due to the simplicity of the method, this is straightforward to implement.

We have applied the method to several quasi-static and dynamic problems which were compared to experimental data or other numerical results from the literature. The agreement between these and our results is good. For the Kalthoff problem, we were able to capture the correct transition from brittleto-ductile failure. The computed shear band and crack pattern agreed well with the experimental results. We were also able to capture the principal failure mechanism of the Nesterenko problem. 


\section{References}

[1] Eurocode 2: Planung von Stahlbeton- und Spannbetontragwerken, Teil 1: Grundlagen und Anwendungsregeln fuer den Hochbau. Ernst und Sohn, Berlin, in Betonkalender 1993, T. II, 1993.

[2] P.M.A. Areias. Finite element technology, damage modeling, contact constraints and fracture analysis. Doutoramento, FEUP - Faculdade de Engenharia da Universidade do Porto, Rua Dr. Roberto Frias s/n 4200-465 Porto, Portugal, 2003. www.fe.up.pt.

[3] P.M.A. Areias, J.M.A. Cesar de Sa, C.A. Conceicao Antonio, J.A.S.A.O. Carneiro, and V.M.P. Teixeira. Strong displacement discontinuity and lagrange multipliers in the analysis of finite displacement fracture problems. Computational Mechanics, in press, 2004.

[4] F. Armero. On the characterization of localized solutions in inelastic solids: an analysis of wave propagation in a softening bar. Computer Methods in Applied Mechanics and Engineering, 191:181-213, 2001.

[5] F. Armero and K. Garikipati. An analysis of strong discontinuities in multiplicative finite strain plasticity and their relation with the numerical simulation of strain localization in solids. International Journal of Solids and Structures, 33(20-22):2863-2885, 1996.

[6] R.C. Batra and R.R. Gummalla. Effect on material and geometric parameters on deformations near the notch-tip of a dynamically loaded prenotched plate. International Journal of fracture, 101:99-140, 2000.

[7] R.C. Batra and N.A. Jaber. Failure mode transition in an impact loaded pre-notched plate with four thermoviscoplastic relations. International Journal of fracture, 110:47-71, 2001.

[8] R.C. Batra, N.A. Jaber, and M.E. Malsbury. Analysis of failure modes in an impact loaded thermoviscoplastic prenotched plate. International Journal of plasticity, 19:139-196, 2003.

[9] R.C. Batra and M.V.S. Ravisankar. Three-dimensional numerical simulation of the kalthoff experiment. International Journal of fracture, 105: 161-186, 2000.

[10] Z. P. Bazant and T. Belytschko. Wave propagation in a strain-softening bar: Exact solution. Journal of Engineering Mechanics-ASCE, 111(3): 381-389, 1985.

[11] T. Belytschko and L.P. Bindemann. Assumed strain stabilization of the 4node quadrilateral with 1-point quadrature for nonlinear problems. Computer Methods in Applied Mechanics and Engineering, 88:311-340, 1991.

[12] T. Belytschko and T. Black. Elastic crack growth in finite elements with minimal remeshing. International Journal for Numerical Methods in Engineering, 45(5):601-620, 1999.

[13] T. Belytschko, H. Chen, J. Xu, and G. Zi. Dynamic crack propagation based on loss of hyperbolicity and a new discontinuous enrichment. International Journal for Numerical Methods in Engineering, 58(12):18731905, 2003. 
[14] T. Belytschko, H-Y Chiang, and E. Plaskacz. High resolution twodimensional shear band computations: imperfections and mesh dependence. Computer Methods in Applied Mechanics and Engineering, 119: $1-15,1994$.

[15] T. Belytschko, J. Fish, and B. Englemann. A finite element method with embedded localization zones. Computer Methods in Applied Mechanics and Engineering, 70:59-89, 1988.

[16] T. Belytschko, Y. Krongauz, D. Organ, M. Fleming, and P. Krysl. Meshless methods: An overview and recent developments. Computer Methods in Applied Mechanics and Engineering, 139:3-47, 1996.

[17] T. Belytschko, W. K. Liu, and B. Moran. Nonlinear Finite Elements for Continua and Structures. John Wiley and Sons, Chichester, 2000.

[18] T. Belytschko and Y.Y. Lu. Element-free galerkin methods for static and dynamic fracture. International Journal of Solids and Structures, 32:2547-2570, 1995.

[19] T. Belytschko, Y.Y. Lu, and L. Gu. Element-free galerkin methods. International Journal for Numerical Methods in Engineering, 37:229-256, 1994.

[20] T. Belytschko and J.S-J. Ong. Hourglass control in linear and nonlinear problems. Computer Methods in Applied Mechanics and Engineering, 43: 251-276, 1984.

[21] T. Belytschko and S.P. Xiao. Stability analysis of particle methods with corrected derivatives. Computers and Mathematics with Applications, 43: 329-350, 2002.

[22] J. Bonet and P. Bhargava. A uniform deformation gradient hexadron element with artificial hourglass control. International Journal for $\mathrm{Nu}$ merical Methods in Engineering, 38:2809-2828, 1995.

[23] O.T. Bruhns, H. Xiao, and A. Meyers. Constitutive inequalities for an isotropic elastic strain-energy function based on Hencky's logarithmic strain tensor. Proceedings of the Royal Society A, London, 457:22072226, 2001.

[24] G. T. Camacho and M. Ortiz. Computational modeling of impact damage in brittle materials. International Journal of Solids and Structures, 33: 2899-2938, 1996.

[25] J.M.A. Cesar de Sa, P.M.A. Areias, and R.M.N. Jorge. Quadrilateral elements for the solution of elasto-plastic finite strain problems. International Journal for Numerical Methods in Engineering, 51:883-917, 2001.

[26] J.S. Chen, S. Yoon, H. Wang, and W.K. Liu. An improved reproducing kernel method for nearly incompressible finite elasticity. Computer Methods in Applied Mechanics and Engineering, 181(1-3):117-145, 2000.

[27] R.J. Clifton, J. Duffy, and K.A. Hartley. On critical conditions for shear band formation at high strain rates. Scripta Metallugrica, 18(5):443-448, 1984.

[28] P. Diez, M. Arroyo, and A. Huerta. Adaptivity based on error estimation for viscoplastic softening materials. Mechanics of Cohesiv-Frictional 
Materials, 5:87-112, 2000.

[29] J. Dolbow and T. Belytschko. Volumetric locking in the element free galerkin method. International Journal for Numerical Methods in Engineering, 46:925-942, 1999.

[30] J. Dolbow, N. Moes, and T. Belytschko. Discontinuous enrichment in finite elements with a partition of unity method. Finite Element Analysis and Design, 36(3):235-260, 2000.

[31] L. Evans and R. Gariepy. Measure Theory and Fine Properties of Functions. CRC Press, New York, 1992.

[32] D.J.F. Ewing and R. Hill. The plastic constraint of v-notched tension bars. Journal of the Mechanics and Physics in Solids, 1967.

[33] M.L. Falk, A. Needleman, and J.R. Rice. A critical evaluation of cohesive zone models of dynamic fracture. Journal of Physics IV 11 (PR5), pages 43-50, 2001.

[34] R.J. Flory. Thermodynamic relations for highly elastic materials. Transactions of the Faraday Society, 57:829-838, 1961.

[35] T.C. Gasser and G.A. Holzapfel. Modeling 3d crack propagation in unreinforced concrete using pufem. Computer Methods in Applied Mechanics and Engineering, 2005.

[36] D.E. Grady. Dissipation in adiabatic shear bands. Mechanics of Materials, 17:289-293, 1994.

[37] A. Gravouil, N. Moes, and T. Belytschko. Non-planar 3D crack growth by the extended finite element and level sets - part ii: Level set update. International Journal for Numerical Methods in Engineering, 53:25692586, 2002.

[38] R.R. Gummalla. Effect of material and geometric parameters on deformations of a dynamically loaded prenotched plate. Master's thesis, Virginia Polytechnical Institute and State University, 1999.

[39] S. Hao, A. Comec, and K.H. Schwalbe. Plastic stress-strain fields and limit loads of a plane strain cracked tensile panel with a mismatched welded joint. International Journal of Solids and Structures, 34:297-311, 1997.

[40] S. Hao, W.K. Liu, and C.T. Chang. Computer implementation of damage models by finite element and meshfree methods. Computer Methods in Applied Mechanics and Engineering, 187(3-4):401-440, 2000.

[41] S. Hao, B. Moran, W.K. Liu, and B. Olson. A hierarchical multi-physics model for design of high toughness steels. Journal of Computer-Aided Materials Design, 10(2):99-142, 2003.

[42] A. Huerta and S. Fernandez-Mendez. Locking in the incompressible limit for the element-free galerkin method. International Journal for Numerical Methods in Engineering, 51:1361-1383, 2001.

[43] A. Huerta, Y. Vidal, and P. Vilon. Pseudo-divergence-free element free galerkin method for incompressible fluid flow. Computer Methods in Applied Mechanics and Engineering, 193(12-14):1119-1136, 2004.

[44] T. J. R. Hughes. Generalization of selective integration procedures to 
anisotropic and nonlinear media. International Journal for Numerical Methods in Engineering, 15(9):1413-1418, 1980.

[45] M. Jirasek. Comparative study on finite elements with embedded discontinuities. Computer Methods in Applied Mechanics and Engineering, 188: 307-330, 2000.

[46] G.R. Johnson and W.H. Cook. A constitutive model and data for metals subjected to large strains, high strain rates, and high temperatures. In Proc. Yth International Symp. on Ballistics, 1983.

[47] J. F. Kalthoff and S. Winkler. Failure mode transition at high rates of shear loading. International Conference on Impact Loading and Dynamic Behavior of Materials, 1:185-195, 1987.

[48] J.F. Kalthoff. Modes of dynamic shear failure in solids. International Journal of Fracture, 101:1-31, 2000.

[49] P. Krysl and T. Belytschko. The element free galerkin method for dynamic propagation of arbitrary 3-D cracks. International Journal for Numerical Methods in Engineering, 44(6):767-800, 1999.

[50] D. Lasry and T. Belytschko. Localization limiters in transient problems. International Journal of Solids and Structures, 24(6):581-597, 1988.

[51] Y.W. Lee, J.C. Woertz, and T. Wierzbicki. Fracture prediction of thin plates under hemi-spherical punch with calibration and experimental verification. International Journal of Mechanical Sciences, 2004.

[52] J. Lemonds and A. Needleman. Finite element analysis of shear localization in rate and temperature dependent solids. Mechanics of Materials, 5:339-361, 1986.

[53] S. Li, W. Hao, and W.K. Liu. Mesh-free simulations of shear banding in large deformation. International Journal of Solids and Structures, 37: 7185-7206, 2000.

[54] S. Li, W. K. Liu, A. J. Rosakis, T. Belytschko, and W. Hao. Mesh free galerkin simulations of dynamic shear band propagation and failure mode transition. International Journal of Solids and Structures, 39:1213-1240, 2002.

[55] R. Lin. Numerical study of consistency of rate constitutive equations with elasticity at finite deformations. International Journal for Numerical Methods in Engineering, 55:1053-1077, 2002.

[56] Y.Y. Lu, T. Belytschko, and M. Tabbara. Element-free galerkin method for wave-propagation and dynamic fracture. Computer Methods in Applied Mechanics and Engineering, 126(1-2):131-153, 1995.

[57] M.A. Meyers, V.F. Nesternko, J.C. LaSalvia, and Q. Xue. Shear localization in dynamic deformation of materials: microstructural evolution and self-organization. Material Science and Engineering, 2001.

[58] K. Minnaar and M. Zhou. An analysis of the dynamic failure resistance of structural metals. Journal Mechanics and Physics of Solids, 46(10): 2155, 21701998.

[59] N Moes and T. Belytschko. Extended finite element method for cohesive crack growth. Engineering Fracture Mechanics, 69:813-834, 2002. 
[60] N. Moes, J. Dolbow, and T. Belytschko. A finite element method for crack growth without remeshing. International Journal for Numerical Methods in Engineering, 46(1):133-150, 1999.

[61] J.C. Nagtegaal, D.M. Parks, and J.R. Rice. On numerically accurate finite element solutions in the fully plastic range. Computer Methods in Applied Mechanics and Engineering, 4:153-177, 1974.

[62] A. Needleman. Dynamics shear band development in plane strain. Journal of Applied Mechanics, 56:1-9, 1989.

[63] A. Needleman and V. Tvergaard. Analysis of brittle-ductile transition under dynamic shear loading. International Journal of Solids and Structures, 32:2571-2590, 1995.

[64] V.F. Nesternko, M.A. Meyers, and T.W. Wright. Self-organization in the initiation of adiabatic shear bands. Acta mater., 1998.

[65] J. Oliver, M. Cervera, and O. Manzoli. Strong discontinuities and continuum plasticity models: the strong discontinuity approach. International Journal of Plasticity, 15:319-351, 1999.

[66] J. Oliver, A.E. Huespe, and E. Samaniego. A study on finite elements for capturing strong discontinuities. International Journal of Numerical Methods in Engineering, 56:1291-1305, 2003.

[67] W.E. Olmstead, S. Nemat-Nasser, and L. Ni. Shear bands as surfaces of discontinuity. Journal of the Mechanics and Physics of Solids, 42: 697-709, 1994.

[68] D. Organ, M. Fleming, T. Terry, and T. Belytschko. Continuous meshless approximations for nonconvex bodies by diffraction and transparency. Computational Mechanics, 18:225-235, 1996.

[69] M. Ortiz, Y. Leroy, and A. Needleman. Finite element method for localized failure analysis. Computer Methods in Applied Mechanics and Engineering, 61(2):189-214, 1987.

[70] P. Perzyna. Fundamental problems in viscoplasticity. In: Recent Advances in Applied Mechanics, Academic Press: New York, 1966.

[71] R. Piltner and R.L. Taylor. A symmetric construction of b-bar functions for linear and non-linear mixed-enhanced finite elements for plane elasticity problems. International Journal for Numerical Methods in Engineering, 44:615-639, 1999.

[72] T. Rabczuk and T. Belytschko. Cracking particles: A simplified meshfree method for arbitrary evolving cracks. International Journal for Numerical Methods in Engineering, 61(13):2316-2343, 2004.

[73] T. Rabczuk and T. Belytschko. Adaptivity for structured meshfree particle methods in 2D and 3D. International Journal for Numerical Methods in Engineering, 63(11):1559-1582, 2005.

[74] T. Rabczuk and T. Belytschko. A three dimensional large deformation meshfree method for arbitrary evolving cracks. Computer Methods in Applied Mechanics and Engineering, in progress.

[75] T. Rabczuk, T. Belytschko, and S.P. Xiao. Stable particle methods based on lagrangian kernels. Computer Methods in Applied Mechanics and En- 
gineering, 193:1035-1063, 2004.

[76] W.D. Reinhardt and R.N. Dubey. Application of objective rates in mechanical modelling of solids. ASME Journal of Applied Mechanics, 118: 692-698, 1996.

[77] E. Samaniego and T. Belytschko. Continuum-discontinuum modelling of shear bands. International Journal for Numerical Methods in Engineering, in Press, 2004.

[78] C.J. Shih, M.A. Meyers, and V.F. Nesterenko. High-strain-rate deformation of granular silicon carbide. Acta mater., 1998.

[79] J. C. Simo and F. Armero. Geometrically non-linear enhanced strain mixed methods and the method of incompatible modes. International Journal for Numerical Methods in Engineering, 33:1413-1449, 1992.

[80] G. Ventura, B. Moran, and T. Belytschko. Dislocations by partition of unity. International Journal for Numerical Methods in Engineering, 62 (11):1463-1487, 2005.

[81] Y. Vidal, P. Villon, and A. Huerta. Locking in the incompressible limit: pseudo-divergence-free element-free galerkin. Revue europeene des elements finis, 11(7-8):869-892, 2002.

[82] D. Wang and J-S. Chen. Locking-free stabilized conforming nodal integration for meshfree mindlind-reissner plate formulation. Computer Methods in Applied Mechanics and Engineering, 193:1065-1083, 2004.

[83] W.M. Wang, L.J. SLuys, and R. deBorst. Viscoplasticity for instabilities due to strain softening and strain-rate softening. International Journal for Numerical Methods in Engineering, 40(20):3839-3864, 1997.

[84] T.W. Wright and J.W. Walter. The asymptotic structure of an adiabatic shear band in antiplane motion. Journal of the Mechanics and Physics of Solids, 44(1):77-97, 1996.

[85] S.P. Xiao and T. Belytschko. Material stability analysis of particle methods. Advances in Computational Mathematics, 2005.

[86] X.-P. Xu and A. Needleman. Numerical simulations of fast crack growth in brittle solids. Journal of the Mechanics and Physics of Solids, 42: 1397-1434, 1994.

[87] Q. Xue, V.F. Nesterenko, and M.A. Meyers. Evaluation of the collapsing thick-walled cylinder technique for shear-band spacing. International Journal of Impact Engineering, 2003.

[88] Q. Yang, A. Mota, and M. Ortiz. A class of variational strain-localization finite elements. International Journal for Numerical Methods in Engineering, 62(8):1013-1037, 2005.

[89] F. Zhou and J.F. Molinari. Dynamic crack propagation with cohesive elements: a methodolgy to address mesh dependence. International Journal for Numerical Methods in Engineering, 59(1):1-24, 2004.

[90] M. Zhou, G. Ravichandran, and A. Rosakis. Dynamically propagating shear bands in impact-loaded prenotched plates-ii. Journal Mechanics Physics and Solids, 44:1007-1032, 1996.

[91] T. Zhu and S.N. Atluri. A modified collocation method and a penalty 
formulation for enforcing the essential boundary conditions in the element free galerkin method. Computational Mechanics, 21(3):211-222, 1998. 